\title{
A Review of Epidemiological Research on Adverse Neurological Effects of Exposure to Ambient Air Pollution
}

\author{
Xiaohui $\mathrm{Xu}^{1 *}$, Sandie Uyen $\mathrm{Ha}^{2}$ and Rakshya Basnet ${ }^{1}$ \\ ${ }^{1}$ Department of Epidemiology and Biostatistics, School of Public Health, Texas A\&M Health Science Center, College Station, \\ TX, USA, ${ }^{2}$ College of Public Health and Health Professions, University of Florida, Gainesville, FL, USA
}

There is a growing body of epidemiological research reporting the neurological effects of ambient air pollution. We examined current evidence, identified the strengths and weaknesses of published epidemiological studies, and suggest future directions for research in this area. Studies were identified through a systematic search of online scientific databases, in addition to a manual search of the reference lists from the identified papers. Despite being a relatively new area of investigation, overall, there is mounting

OPEN ACCESS

Edited by:

André F. S. Amaral,

Imperial College London, UK

Reviewed by:

Vito llacqua,

Environmental Protection

Agency, USA

Tamara Schikowski,

Leibniz Institute of Environmental

Medicine (LG), Germany

*Correspondence: Xiaohui Xu

xiaohui.xu@sph.tamhsc.edu

Specialty section: This article was submitted to Epidemiology,

a section of the journal

Frontiers in Public Health

Received: 13 January 2016

Accepted: 19 July 2016

Published: 05 August 2016

Citation:

Xu X, Ha SU and Basnet R (2016)

A Review of Epidemiological

Research on Adverse Neurological Effects of Exposure to Ambient Air

Pollution.

Front. Public Health 4:157. doi: 10.3389/fpubh.2016.00157 evidence implicating adverse effects of air pollution on neurobehavioral function in both adults and children. Further research is needed to expand our understanding of these relationships, including improvement in the accuracy of exposure assessments; focusing on specific toxicants and their relationships to specific health endpoints, such as neurodevelopmental disorders and neurodegenerative diseases; investigating the combined neurological effects of multiple air pollutants; and further exploration of genetic susceptibility for neurotoxicity of air pollution. In order to achieve these goals collaborative efforts are needed from multidisciplinary teams, including experts in toxicology, biostatistics, geographical science, epidemiology, and neurology.

\section{Keywords: air pollution, neurotoxicity, cognitive function, particulate matter, neurological effects, brain}

\section{INTRODUCTION}

Over the past 30 years, extensive evidence has shown that air pollution affects cardiovascular and respiratory morbidity and mortality in both adults and children across the world (1-4). Air pollution has also been consistently and widely associated with elevated risks of adverse pregnancy outcomes such low birth weight $(5,6)$, preterm delivery $(7,8)$, intrauterine growth retardation $(9,10)$, and birth defects $(10,11)$. More recently, there have been studies examining the link between air pollution and adverse neurological outcomes.

Incidence rates of diseases of the nervous system, such as neurodegenerative diseases in adults and neurodevelopmental disorders in children, have increased over the past years $(12,13)$. Fox et al. conducted a cumulative risk assessment for 40 ambient hazardous air pollutants (HAPs) based on either the single-effect toxicological data from the U.S Environmental Protection Agency (EPA) or their own multiple-effect toxicological database. The cumulative risk assessment of 40 HAPs revealed that neurological effects ranked the 2nd out of 17 health effects - only after respiratory effects - regardless of the data sources (14). The nervous system, particularly the central nervous system (CNS), is vulnerable to oxidative stress because it has high metabolic demands, high energy use, 
widespread axonal and dendritic networks, high cellular content of lipids and proteins, and low levels of endogenous scavengers, such as vitamin $\mathrm{C}$ and superoxide dismutase, which, to some extent, may be due to the CNS being isolated (15). The CNS in a child could be especially susceptible to oxidative stress from environmental toxicants because of its underdeveloped barrier and a wide time window of conformation. In fact, a systematic review by Landrigan et al. has found that exposure to pollution in early life has special implications in neurogenerative effects later in life (16). Thus, because of the ubiquity of toxicants present in air, potential neurological effects need to be evaluated.

Air pollution is a multifaceted environmental toxicant that comprises a diverse mixture of particulate matters (PMs), including organic components and metals, and gases, such as nitrogen oxides, sulfur oxides, and ozone. It can generate reactive oxygen species, deplete endogenous antioxidants, alter mitochondrial functions, and produce oxidative damage to lipids and DNA (17). Inflammation and oxidative stress have been recognized as the main potential mechanisms through which air pollution causes damage to cardiovascular and respiratory systems (18-20). Thus, it is logical to hypothesize that air pollution could also cause damage to the nervous system through oxidative stress pathways. Oberdorster and Utell first raised the concern that the brain may be targeted by ultrafine PM (21). Since then, several population-based studies have been conducted to evaluate the adverse neurologic effects of exposure to ambient air pollution.

The purpose of this review is to systematically examine population-based studies evaluating the relationship between air pollution and neurological outcomes to determine if there is sufficient evidence to suggest a causal link and to identify knowledge gaps to help guide new research efforts.

\section{MATERIALS AND METHODS}

We searched all publications included in the electronic databases of PubMed (from 1966 to present, National Library of Medicine, Bethesda, MD, USA), Google Scholar (Google Inc., Mountain View, CA, USA), and the Institute of Scientific Information Web of Knowledge (from 1966-present, Thompson Scientific, Philadelphia, PA, USA). We used different combinations of MeSH headings of "air pollution," "air pollutant," "PM," "HAP," or "AQS" with any of the following: "cognitive," "cognition," "nervous system diseases," "neurotoxicity syndromes," "neurodegenerative diseases," "neurodevelopmental diseases," "Alzheimer," "Parkinson," "amyotrophic lateral sclerosis (ALS)," "Huntington," "autism," "attention-deficit hyperactivity disorder (ADHD)," and "learning disability." Furthermore, we also reviewed the reference lists of the identified papers and manually searched for additional publications. All research articles pertaining to studying health effects of ambient air pollution on cognitive performance, neurological symptoms, and neurobehavioral disorders were selected for a full review. In addition, we included abstracts of conference presentations if the information provided was adequate to make an evaluation. We excluded studies that were not published in English or those that were not original studies. In addition, we excluded research on stroke as the relationship between air pollution and cardiovascular diseases have been extensively studied. Because of our focus on ambient air pollution, we also excluded studies that investigated air pollutants related to occupational, accidental exposure, or indoor generated pollutants. Since neurological effects of lead exposures are well-established, we did not include studies pertaining to this topic. The search was last updated in May 2016.

\section{RESULTS}

Figure 1 illustrates the study inclusion and exclusion process. Initial search from three large search engines yielded more than 13,000 entries, yielding 689 unduplicated articles. After applying inclusion/exclusion criteria, 66 studies remained in this review. Among them, one study examined both children and adult (22), and the other study examined multiple outcomes (23). Despite being a relatively new area of investigation, there is mounting evidence implicating adverse effects of air pollution on the nervous system in both adults and children. Ambient air pollution may be associated with biological changes in the brain, such as change in brain activity, increase in inflammatory reaction, and pathological changes in brain tissues in children and/or adults $(22,24,25)$. Moreover, studies consistently showed that ambient air pollution, particularly traffic-related air pollution (TRAP), is associated with various adverse neurological health effects, including decreased neurocognitive abilities, such as memory and motor responses; nervous system sequelae, such as fatigues, headache, and inability to concentrate; and neurological disorders, such as Alzheimer's, Parkinson's, ADHD, and autism. In adults, a total of 26 studies have been identified for a full review. Among them, 4 studies on brain histology and activity (24-27); 13 studies examined the health effects of ambient or traffic air pollution on cognitive functions (28-40); 7 study examined the effects of ambient air pollutants on neurodegenerative diseases (41-47); and 1 on neurological symptoms, such as headache and fatigue and trouble concentrating (48). In children, 41 studies have been selected for a full review: 3 studied brain histological anomalies $(22,23,49)$; a total of 24 studies have investigated the effects of prenatal and/or postnatal exposure to air pollution on neurodevelopment and cognitive functions among children (23, 50-72); and 15 studies investigated the effects of air pollution on risks of neurodevelopmental disorders, such as ADHD (73-75) and autism (76-87). These studies are individually summarized and discussed below and in Tables $\mathbf{1}$ and $\mathbf{2}$ for adults and children, respectively. We also critically evaluate potential epidemiological limitations regarding the published studies we reviewed in this area, including measurement of exposures, study design, confounding, and biases in the Section "Discussion."

\section{Air Pollution and Neurotoxicity in Adults Effects on Brain Biology and Histology}

Histological and biological changes in the CNS in human after exposure to ambient air pollution have rarely been studied, perhaps due to the cost associated with complex study procedures. Two studies examined the association between air pollution and human brain inflammation $(24,26)$, and two controlled human exposure studies investigated the effect of exposure to diesel exhaust on the CNS $(25,27)$. The changes of expression 


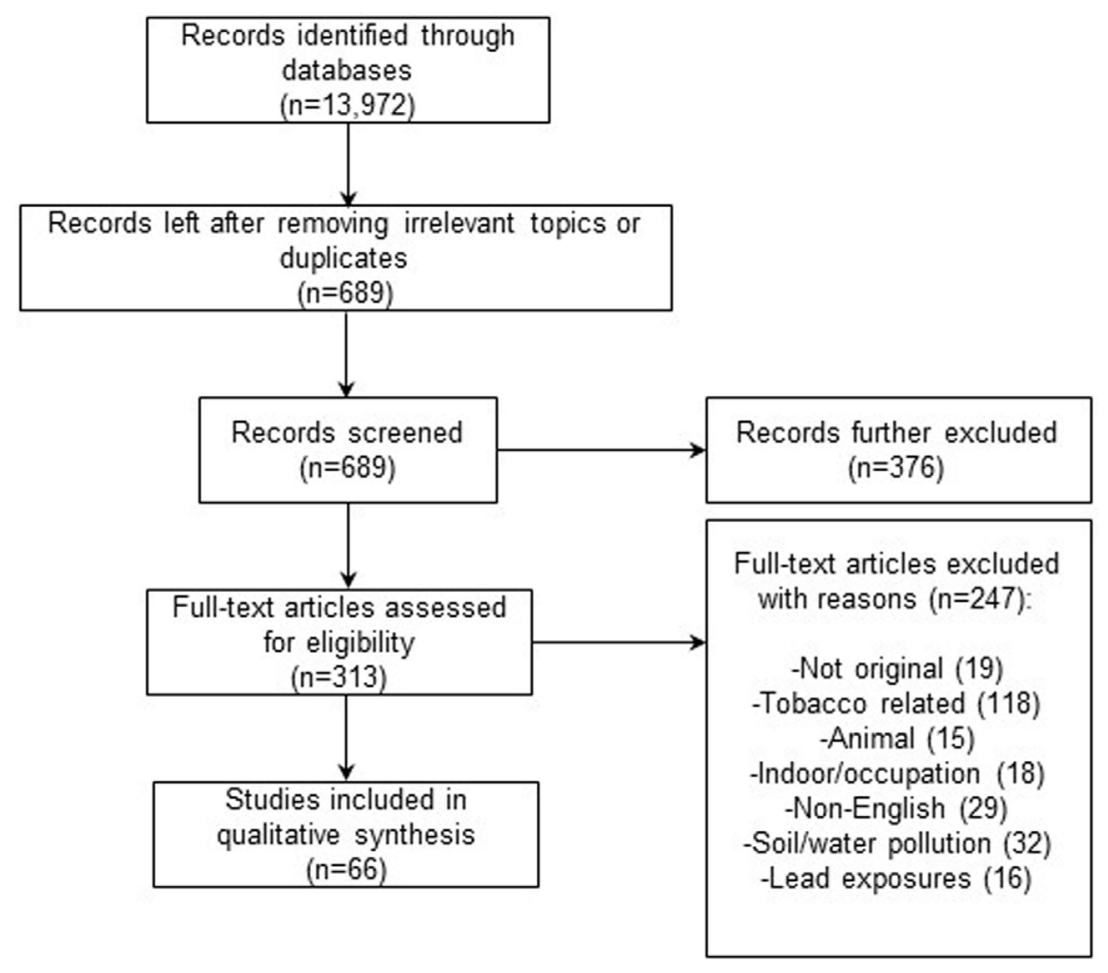

FIGURE 1 | Study selection.

of cyclooxygenase-2 (COX2) and accumulation of the 42-amino acid form of $\beta$-amyloid (A $\beta 42)$, which are two common inflammatory markers, were examined in human brain samples from lifelong residents of large cities with severe air pollution (exposed) and control subjects from small cities with low air pollution levels (unexposed) in Mexico. The studies reported significantly higher COX2 expression and greater neuronal and astrocytic accumulation of $A \beta 42$ among residents of cities with severe air pollution compared to residents in low air pollution cities (24, 26). The authors contend that high COX2 expression and A $\beta 42$ accumulation are characteristics of neurological disorders, such as Alzheimer's disease, suggesting that air pollution may increase the risk of neurological disorders. Despite significant findings and the important contribution to the field as one of the few studies investigating the link between air pollution and brain histology, the results of these studies need to be interpreted with caution because of some limitations. Specifically, these studies have very small, convenient sample sizes of 19 and 59, respectively. The low sample sizes threaten the studies' validity and prevent them from making generalization. People who were selected to be in the studies may not be representative of the whole population in high and low air pollution exposure areas under study. Second and most importantly, the studies do not have a clearly defined independent variable to be tested. They examined two groups of people from high and low pollution areas; however, high and low pollution were not objectively defined, making it highly subjected to exposure misclassification since personal exposure could vary from person to person despite living in the same area. In other words, a person living in a low exposed city may have higher personal exposure level. In addition, the analyses were not adjusted for important factors besides pollution that could have explained the differences in brain histology found in the studies.

In an attempt to minimize threats to validity, such as confounding and misclassification between comparison groups, Cruts et al. (25) conducted a double-blind randomized crossover study to examine the relationship between exposure to dilute diesel exhaust and changes in brain activity measured by quantitative electroencephalography. Diesel exhaust was delivered for $30 \mathrm{~min}$ by an engine which produced $300 \mu \mathrm{g} / \mathrm{m}^{3}$ of suspended particles, $1.6 \mathrm{ppm}$ nitrogen oxide, $4.5 \mathrm{ppm}$ nitric oxide, $7.5 \mathrm{ppm}$ carbon monoxide, and $7.5 \mathrm{ppm}$ total hydrocarbon. The authors found a significant increase in median power frequency in the frontal cortex after $30 \mathrm{~min}$ of exposure to diesel exhaust. This study suggests that diesel exposure can lead to a general cortical stress response (25). However, Cliff et al. used the same study design to examine the acute effects of diesel exhaust exposure on biomarkers of systemic and CNS inflammation. No significant effects were observed among 27 healthy adults (27). In these two studies, confounding is not likely a major issue because the comparison groups are the same subjects. However, again, it has limited generalizability due to a small sample size.

Overall, despite there are numerous studies showing exposure to air pollution can increase neuroinflammation in animal studies (88-91), evidence among human is still relatively limited and inconsistent. The need to examine brain tissues and activities prevents human studies from being abundant. Additionally, the 


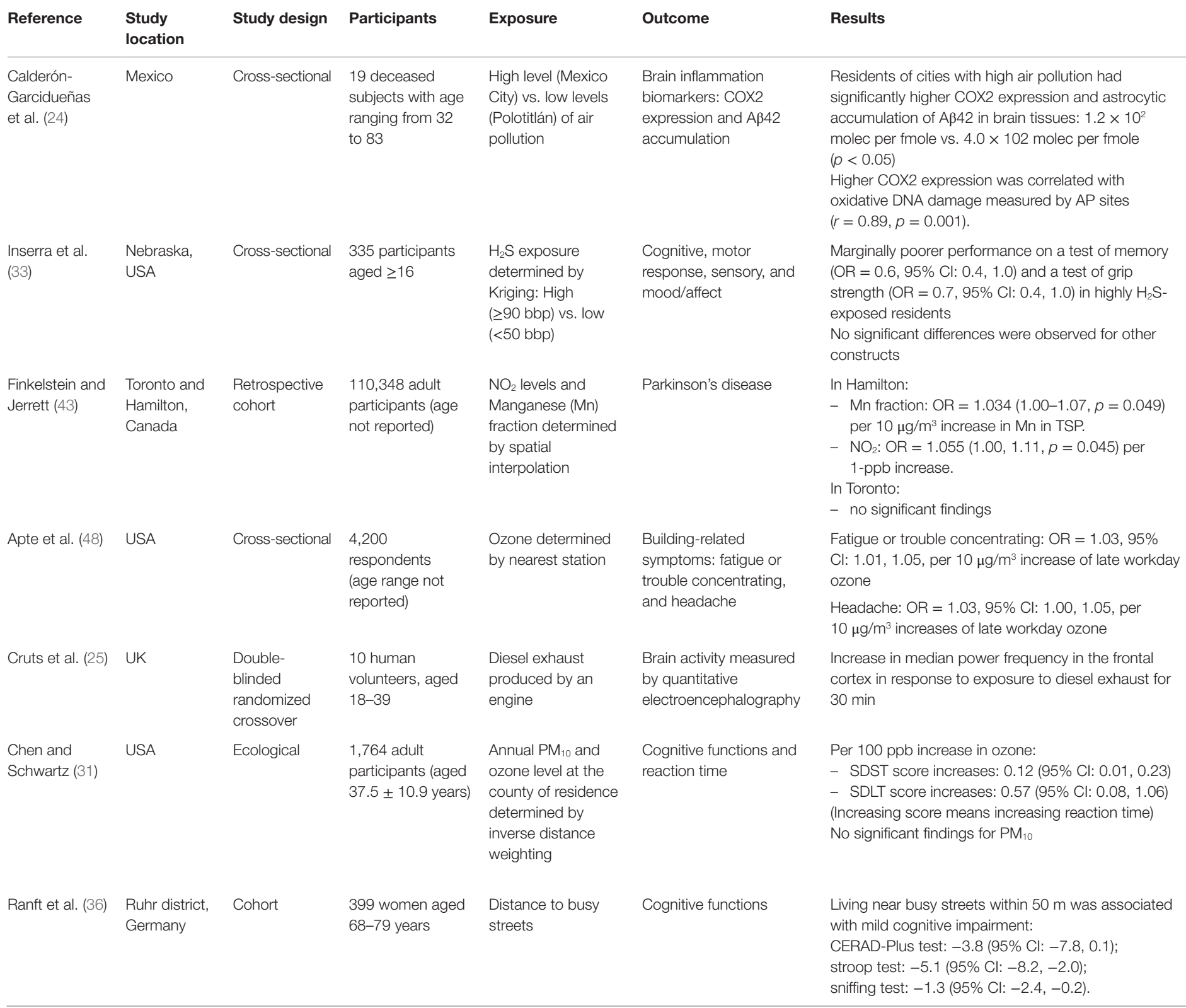

N/A

Age, sex, and testing language

Year of birth, sex

Season, sex age, smoking status, environmental sensitivities, relative humidity, and thermal exposure

N/A

Age, sex, race, education, employment status, smoking status, alcohol consumption, family size, annual family income, poverty-income ratio, and type of residence (urban/rural), CVD risk factors

Age, years live in area, smoking status, environmental quality, indoor pollution, history of chronic diseases, depression, education 


\begin{tabular}{|c|c|c|c|c|c|c|c|}
\hline Reference & $\begin{array}{l}\text { Study } \\
\text { location }\end{array}$ & Study design & Participants & Exposure & Outcome & Results & Covariates \\
\hline $\begin{array}{l}\text { Calderón- } \\
\text { Garcidueñas } \\
\text { et al. (22) }\end{array}$ & Mexico & Cross-sectional & $\begin{array}{l}44 \text { adults and } \\
\text { children aged } \\
2-40 \text { years }\end{array}$ & $\begin{array}{l}\text { High (Mexico City)/ } \\
\text { low (Polotitlan) levels } \\
\text { of air pollution }\end{array}$ & $\begin{array}{l}\text { The University of } \\
\text { Pennsylvania Smell } \\
\text { Identification Test (UPSIT) } \\
\text { score } \\
\text { Olfactory bulbs (OB) } \\
\text { pathology }\end{array}$ & $\begin{array}{l}\text { MC subjects had significantly lower UPSIT scores: } \\
34.24 \pm 0.42 \text { vs. controls } 35.7 \pm 0.40, p=0.03 \\
\text { The exposed groups exhibited remarkable } \\
\text { immunoreactivity to A } \beta 42 \text { and } \alpha \text {-synuclein, and OB } \\
\text { endothelial hyperplasia, and neuronal accumulation } \\
\text { of particles }\end{array}$ & N/A \\
\hline $\begin{array}{l}\text { Power et al. } \\
\text { (35) }\end{array}$ & USA & $\begin{array}{l}\text { Retrospective } \\
\text { cohort }\end{array}$ & $\begin{array}{l}680 \text { men } \\
\text { (mean } \pm \text { SD, } \\
71 \pm 7 \text { years of } \\
\text { age) }\end{array}$ & $\begin{array}{l}\text { Black carbon } \\
\text { determined by } \\
\text { land-use regression } \\
\text { models }\end{array}$ & Cognitive functions & $\begin{array}{l}\text { Low-mini-mental status examination score }(<25) \text { : } \\
\text { OR }=1.3(1.1-1.6) \text { for each doubling in black carbon } \\
\text { concentration } \\
\text { Each doubling in black carbon concentration was } \\
\text { also associated with a } 0.054 \text { SD lower cognitive test } \\
\text { score }(95 \% \mathrm{Cl}:-0.103,-0.006)\end{array}$ & $\begin{array}{l}\text { Age, education, first language, } \\
\text { computer experience, physical } \\
\text { activity, alcohol consumption, } \\
\text { diabetes, dark fish consumption, } \\
\text { percentage of residential } \\
\text { census tract that is non-white, } \\
\text { percentage of residential } \\
\text { census tract adults with a } \\
\text { college degree, indicator for } \\
\text { first cognitive assessment, and } \\
\text { indicator for part-time resident }\end{array}$ \\
\hline $\begin{array}{l}\text { Wellenius et al. } \\
\text { (39) }\end{array}$ & USA & $\begin{array}{l}\text { Retrospective } \\
\text { cohort }\end{array}$ & $\begin{array}{l}765 \text { adults seniors } \\
\text { aged } \geq 65 \text { years }\end{array}$ & $\begin{array}{l}\text { Distance to major } \\
\text { road way }\end{array}$ & Cognitive functions & $\begin{array}{l}\text { An interquartile range decrease }(851.2 \mathrm{~m}) \text { in } \\
\text { residential distance to major roadway is associated } \\
\text { with decreased scores for immediate [-0.6 }(-1.1 \text { to } \\
-0.1)] \text { and delayed }[-0.4(-0.7 \text { to }-0.1)] \text { recall, letter } \\
{[-1.4(-2.7 \text { to }-0.2)] \text { and category influency }[-0.7} \\
(-1.1 \text { to }-0.3)] \text {, and trail making test scores [10.5 } \\
(4.0-17.1)]\end{array}$ & $\begin{array}{l}\text { Age, sex, race, history of } \\
\text { stroke, history of smoking, } \\
\text { education, visit number, body } \\
\text { mass index, physical activity, } \\
\text { household income, percentage } \\
\text { of neighborhood population } \\
\text { that is non-white, and } \\
\text { percentage of neighborhood } \\
\text { population with a college } \\
\text { degree or above }\end{array}$ \\
\hline $\begin{array}{l}\text { Weuve et al. } \\
(40)\end{array}$ & USA & Cohort & $\begin{array}{l}19,409 \text { US } \\
\text { women aged } \\
70-81 \text { years }\end{array}$ & $\begin{array}{l}\mathrm{PM}_{2.5-10} \text {, and } \\
\mathrm{PM}_{2.5} \text { determined } \\
\text { by USEPA } \\
\text { spatiotemporal } \\
\text { smoothing models }\end{array}$ & $\begin{array}{l}\text { Global cognitive scores } \\
\text { measured by Telephone } \\
\text { Interview for Cognitive } \\
\text { Status (TICS) }\end{array}$ & $\begin{array}{l}\text { For long-term exposure ( } 7-14 \text { years): } \\
\mathrm{PM}_{2.5-10} \text { exposure: } \\
\text { - The 2-year change in standardized cognitive } \\
\text { scores for each } 10 \mu \mathrm{g} / \mathrm{m}^{3} \text { increment in } \mathrm{PM}_{2.5-10} \\
\text { exposure is: }-0.020,95 \% \mathrm{Cl} \text { : }-0.032,-0.008 \\
\mathrm{PM}_{2.5} \text { exposure: } \\
\text { - The 2-year change in standardized cognitive } \\
\text { scores for each } 10 \mu \mathrm{g} / \mathrm{m}_{3} \text { increment in } \mathrm{PM}_{2.5} \\
\text { exposure is: }-0.018(95 \% \mathrm{Cl}:-0.035,-0.002)\end{array}$ & $\begin{array}{l}\text { Age, education, husband's } \\
\text { education, long-term physical } \\
\text { activity, and long-term alcohol } \\
\text { consumption }\end{array}$ \\
\hline $\begin{array}{l}\text { Calderón- } \\
\text { Garcidueñas } \\
\text { et al. (26) }\end{array}$ & Mexico & Cross-sectional & $\begin{array}{l}59 \text { participants } \\
\text { (age } \\
33.06 \pm 4.8 \text { years) }\end{array}$ & $\begin{array}{l}\text { Air pollution levels } \\
\text { High: Mexico city } \\
\text { Low: Tlaxcala and } \\
\text { Veracruz }\end{array}$ & $\begin{array}{l}\text { Brain inflammation } \\
\text { markers: COX2 and IL1 } \beta\end{array}$ & $\begin{array}{l}\text { COX2 in the frontal cortex was significantly } \\
\text { elevated in Mexico City subjects vs. control, } \\
p=0.008 \text {. Exposed subjects also had higher IL } 1 \beta \\
\text { expression in the frontal cortex, } p=0.0002 \text {. COX2 } \\
\text { in olfactory bulb was significantly elevated vs. } \\
\text { control, } p=0.005\end{array}$ & N/A \\
\hline
\end{tabular}


Reference Study

Study design

Exposure

Outcome

Results

Covariates

Loop et al. (34) USA

Cohort

20,150 men and

Satellite-derived

Satellite-derived
estimate of 1-year

women aged

mean $\mathrm{PM}_{2.5}$

Cognitive status

$\sim 64 \pm 9.2$ years

concentration

telephone using th

Six-Item Screener (SIS)

A $10-\mathrm{mg} / \mathrm{m}^{3}$ increase in $\mathrm{PM}_{2.5}$ concentration was not reliably associated with an increased odd of incident impairment $(\mathrm{OR}=1.2695 \% \mathrm{Cl}: 0.97,1.64)$.

Age, sex, race, region, income, education level, depressive symptoms, smoking status, alcohol use, level of exercise, temperature, season, incident stroke, length of follow-up

Ailshire and USA

Crimmins (29)

\section{Chang et al. Taiwan}

(42)

Los Angeles,

USA

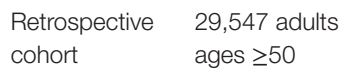

13,996 men and
women aged

50 years or older

Average $\mathrm{PM}_{2.5}$

concentration from based on the Telephone

each monitoring

station were

weighted using

the inverse of the

distance

Yearly average

$\mathrm{NO}_{2}$ and $\mathrm{CO}$

concentrations

measured at the

monitoring stations

Interview for Cognitive

Status

Older adults living in areas with higher $\mathrm{PM}_{25}$

concentrations (highest quartile) had worse cognitive function ( $\beta=-0.26,95 \% \mathrm{Cl}:-0.47,-0.05$ ).

\section{Average $\mathrm{O}_{3}$,}

$\mathrm{PM}_{2.5}$, and $\mathrm{NO}_{2}$

concentration

from 2000-2006

estimated using

monitoring data and

inverse distance

weighing

\begin{tabular}{|c|c|c|c|}
\hline $\begin{array}{l}\text { Heydarpour } \\
\text { et al. (44) }\end{array}$ & Tehran, Iran & $\begin{array}{l}\text { Cross- } \\
\text { sectional } \\
\text { (cluster } \\
\text { analysis) }\end{array}$ & $\begin{array}{l}2,188 \text { multiple } \\
\text { sclerosis among } \\
8.2 \text { million } \\
\text { background } \\
\text { population }\end{array}$ \\
\hline $\begin{array}{l}\text { Tonne et al. } \\
\text { (37) }\end{array}$ & $\begin{array}{l}\text { London, } \\
\text { England }\end{array}$ & Cohort & $\begin{array}{l}2867 \text { adults ages } \\
66 \pm 6 \text { years }\end{array}$ \\
\hline
\end{tabular}

$\mathrm{PM}_{10}, \mathrm{SO}_{2}, \mathrm{NO}, \mathrm{NO}, \quad$ Multiple sclerosis (MS)

$\mathrm{NO}_{x}$ concentration diagnosis meeting

during the year McDonald's criteria

(n)

using LUR

(37) England
$66 \pm 6$ years
Dementia assessed

using International

Classification of Disease

Ninth Revision, Clinical

Modification (ICD-9-CM)

Global cognition and

six domains of cognitive

function using a battery

of tests

Decline in reasoning, memory, and phonemic and semantic fluency
The adjusted hazard ratios (HRs) of dementia for all participants in Q2, Q3, and Q4 compared to Q1 were as follows: for $\mathrm{NO}_{2}: 1.10$ (95\% Cl: $\left.0.96,1.26\right), 1.01$ (95\% Cl: $0.87,1.17)$, and $1.54(95 \% \mathrm{Cl}, 1.34,1.77)$; and for CO: 1.07 (95\% Cl: 0.92, 1.25), $1.37(95 \% \mathrm{Cl}$ :

$1.19,1.58)$, and 1.61 (95\% Cl: $1.39,1.85)$

Increasing exposure to $\mathrm{PM}_{2.5}$ was associated with lower verbal learning $\left(\beta=-0.32\right.$ per $10 \mu \mathrm{g} / \mathrm{m}^{3}$. 95\% Cl: $-0.63,0.00)$

$\mathrm{NO} 2>20 \mathrm{ppb}$ was associated with lower logical memory $[\beta=-0.62(-1.35,0.11)]$

O3 exposure above $49 \mathrm{ppb}$ was associated with lower executive function $[\beta=-0.66(-1.35,0.03)]$

Prevalent MS cases had a clustered pattern in Tehran. A significant difference in exposure to $\mathrm{PM}_{10}$, $\mathrm{SO}_{2}, \mathrm{NO}_{2}$, and $\mathrm{NO} \times(p<0.001)$ was observed in MS cases compared with controls

Higher $\mathrm{PM}_{2.5}$ of $1.1 \mu \mathrm{g} / \mathrm{m}^{3}$ (lag 4) was associated with a 0.03 (95\% Cl: -0.06 to 0.002) 5-year decline
Average $\mathrm{PM}_{10}$ and $\mathrm{PM}_{2.5}$ concentration estimated by

dispersion model at

home address in standardized memory score and a $0.04(-0.07$ to -0.01 ) decline when restricted to participants remaining in London between study waves

Age, sex, and race/ethnicity, educational attainment, current employment status, income, smoking behavior, census tractlevel proportion of residents aged 25 years or older without a high school degree and median household income

Age, sex, monthly income,

diabetes, hypertension, chronic obstructive pulmonary disease, alcoholism, and urbanization

Age, gender, race, education, income, study, and mood

N/A

Age, sex, ethnicity, marital status, educational achievement, socioeconomic position, smoking status, alcohol use, frequency of fruit and vegetable consumption, physical activity, blood pressure, serum cholesterol levels,

prevalence of stroke, coronary

heart disease and diabetes, and frequency of depressive symptoms 


\begin{tabular}{|c|c|c|c|c|c|c|c|}
\hline Reference & $\begin{array}{l}\text { Study } \\
\text { location }\end{array}$ & Study design & Participants & Exposure & Outcome & Results & Covariates \\
\hline $\begin{array}{l}\text { Ailshire and } \\
\text { Clarke (28) }\end{array}$ & USA & Cohort & $\begin{array}{l}780 \text { men and } \\
\text { women aged } 55 \\
\text { and older }\end{array}$ & $\begin{array}{l}\text { Annual } \mathrm{PM}_{2.5} \\
\text { levels based on air } \\
\text { monitoring data }\end{array}$ & $\begin{array}{l}\text { Cognitive function } \\
\text { assessed with an } \\
\text { abbreviated form of the } \\
\text { Short Portable Mental } \\
\text { Status Questionnaire } \\
\text { (SPMSQ) }\end{array}$ & $\begin{array}{l}\text { Those living in areas with greater exposure to } \mathrm{PM}_{2.5} \\
\text { had an error rate } 1.5 \text { times greater than those } \\
\text { exposed to lower } \mathrm{PM}_{2.5} \text { concentrations (IRR }=1.53 \text {; } \\
95 \% \mathrm{Cl}: 1.02,2.30 \text { for } 10 \mu \mathrm{g} / \mathrm{m}^{3} \text { increment) }\end{array}$ & $\begin{array}{l}\text { Age, gender, race, education, } \\
\text { income, marital status, } \\
\text { employment status, and residential } \\
\text { tenure }\end{array}$ \\
\hline $\begin{array}{l}\text { Bowler et al. } \\
\text { (30) }\end{array}$ & Ohio, USA & Cross-sectional & $\begin{array}{l}186 \text { adults } \\
56 \pm 10.8 \text { years }\end{array}$ & $\begin{array}{l}\text { Air borne } \\
\text { manganese } \\
\text { (Mn) estimated } \\
\text { using U.S. EPA's } \\
\text { AERMOD dispersion } \\
\text { model }\end{array}$ & $\begin{array}{l}\text { Cognitive function(s) } \\
\text { assessed by a battery } \\
\text { of tests }\end{array}$ & $\begin{array}{l}\text { Significant relationships }(p<0.05) \text { were found } \\
\text { between } M n \text { exposure and performance on working } \\
\text { and visuospatial memory }(e . g ., \text { Rey-O Immediate } \\
\beta=-0.19 \text {, Rey-O Delayed } \beta=-0.16 \text { ) and verbal } \\
\text { skills (e.g., similarities } \beta=-0.19)\end{array}$ & $\begin{array}{l}\text { Education, town of residence, } \\
\text { age, income, ethnicity, years of } \\
\text { residence, BMl, employment } \\
\text { status, sex, race }\end{array}$ \\
\hline Jung et al. (45) & Taiwan & Cohort & $\begin{array}{l}95,690 \text { individuals } \\
\text { ages } \geq 65\end{array}$ & $\begin{array}{l}\mathrm{O}_{3} \text { and } \mathrm{PM}_{2.5} \\
\text { exposure estimated } \\
\text { using inverse } \\
\text { distance weighting }\end{array}$ & $\begin{array}{l}\text { Newly diagnosed } \\
\text { Alzheimer's disease } \\
\text { assessed by ICD-9-CM }\end{array}$ & $\begin{array}{l}\text { The adjusted } \mathrm{HR} \text { for } \mathrm{AD} \text { was weakly associated } \\
\text { with a raised concentration in O3 at baseline per } \\
\text { increase of } 9.63 \mathrm{ppb} \text { (adjusted } \mathrm{HR}: 1.06) \text {. There was } \\
\text { a } 211 \% \text { increase } \mathrm{AD} \text { risk per increase of } 10.91 \mathrm{ppb} \\
\text { in } \mathrm{O}_{3} \text { over the follow-up period ( } 95 \% \mathrm{Cl}: 2.92 \text {, } \\
3.33 \text { ), and a } 138 \% \text { increase } \mathrm{AD} \text { risk per increase } \\
\text { of } 4.34 \mu \mathrm{\mu g} / \mathrm{m}^{3} \text { in } \mathrm{PM} \mathrm{M}_{2.5} \text { over the follow-up period } \\
(95 \% \mathrm{Cl}: 2.21,2.56)\end{array}$ & $\begin{array}{l}\text { Age, gender, income, and other } \\
\text { comorbidities (e.g., diabetes, } \\
\text { hypertension, myocardial } \\
\text { infarction, angina pectoris, stroke, } \\
\text { peripheral artery disease, asthma, } \\
\text { COPD) }\end{array}$ \\
\hline $\begin{array}{l}\text { Kalkbrenner } \\
\text { et al. (83) }\end{array}$ & $\begin{array}{l}\text { North } \\
\text { Carolina and } \\
\text { California, } \\
\text { USA }\end{array}$ & Case-control & $\begin{array}{l}979 \text { cases and } \\
14,666 \text { controls }\end{array}$ & $\mathrm{PM}_{10}$ exposure & $\begin{array}{l}\text { Autism Spectrum } \\
\text { Disorder }\end{array}$ & $\begin{array}{l}\mathrm{PM}_{10} \text { exposure at the third trimester was associated } \\
\text { with autism }(\mathrm{OR}=1.36,95 \% \mathrm{Cl}: 1.13-1.63)\end{array}$ & $\begin{array}{l}\text { Year, state, maternal education } \\
\text { and age, race/ethnicity, } \\
\text { neighborhood-level urbanization } \\
\text { and median household income, } \\
\text { and seasonality }\end{array}$ \\
\hline $\begin{array}{l}\text { Malek et al. } \\
(46)\end{array}$ & $\begin{array}{l}\text { Pennsylvania, } \\
\text { USA }\end{array}$ & Case-control & $\begin{array}{l}51 \text { cases and } 51 \\
\text { matched controls } \\
\text { ages' } \geq 18 \\
\text { (matched on age } \\
\text { of onset, race, } \\
\text { sex) }\end{array}$ & $\begin{array}{l}\text { Hazardous air } \\
\text { pollutants (HAPs) } \\
\text { exposures on } \\
\text { census-tract level } \\
\text { estimated by U.S. } \\
\text { Environmental } \\
\text { Protection Agency } \\
\text { (EPA) National- } \\
\text { Scale Air Toxics } \\
\text { Assessment (NATA) }\end{array}$ & $\begin{array}{l}\text { Amyotrophic lateral } \\
\text { sclerosis (ALS) }\end{array}$ & $\begin{array}{l}\text { Residential exposure to aromatic solvents significantly } \\
\text { elevated the risk of ALS among cases compared to } \\
\text { controls in } 2002 \text { (OR } 1 / 45.03,95 \% \mathrm{Cl} \text { 1.29-19.53) } \\
\text { and } 1999 \text { (OR } 1 / 44.27,95 \% \mathrm{Cl} \text { 1.09-16.79) }\end{array}$ & $\begin{array}{l}\text { Education, smoking, and other } \\
\text { exposure groups (metals, } \\
\text { pesticides, other HAPs) }\end{array}$ \\
\hline $\begin{array}{l}\text { Angelici et al. } \\
(41)\end{array}$ & $\begin{array}{l}\text { Lombardy } \\
\text { region, Italy }\end{array}$ & Cohort & $\begin{array}{l}8287 \text { hospital } \\
\text { admissions (linked } \\
\text { with MS) in } 107 \\
\text { hospitals between } \\
\text { 2001-2009 }\end{array}$ & $\begin{array}{l}\text { Exposure to } \\
\text { particulate matter }\end{array}$ & $\begin{array}{l}\text { Multiple sclerosis hospital } \\
\text { admission }\end{array}$ & $\begin{array}{l}\text { A higher RR of hospital admission for MS relapse was } \\
\text { associated with exposure to } \mathrm{PM}_{10} \text { at different time } \\
\text { intervals. Hospital admission for MS increased } 42 \% \\
\text { ( } 95 \% \mathrm{Cl}: 1.39-1.45 \text { ) on the days preceded by } 1 \text { week } \\
\text { with PM10 levels in the highest quartile. The } p \text {-value } \\
\text { for trend across quartiles was }<0.001\end{array}$ & $\begin{array}{l}\text { Number of MS hospital } \\
\text { admissions, } \mathrm{PM}_{10} \text { concentration, } \\
\text { day of the week on the day of } \\
\text { admission, seasonality, day-off, } \\
\text { resident population in each area, } \\
\text { smoothing spline functions of time, } \\
\text { day of the week and smoothing } \\
\text { splines of 3-day lags of average } \\
\text { temperature with } 3 \mathrm{df}\end{array}$ \\
\hline
\end{tabular}


TABLE 1 | Continued

\begin{tabular}{|c|c|c|c|c|c|c|c|}
\hline Reference & $\begin{array}{l}\text { Study } \\
\text { location }\end{array}$ & Study design & Participants & Exposure & Outcome & Results & Covariates \\
\hline Cliff et al. (27) & $\begin{array}{l}\text { Vancouver, } \\
\text { Canada }\end{array}$ & $\begin{array}{l}\text { Controlled } \\
\text { blinded } \\
\text { crossover }\end{array}$ & $\begin{array}{l}27 \text { healthy adults } \\
\text { aged } 19-49 \text { years }\end{array}$ & $\begin{array}{l}\text { Exposure to air } \\
\text { pollution }\end{array}$ & $\begin{array}{l}\text { Biomarkers of systemic } \\
\text { and CNS inflammation }\end{array}$ & $\begin{array}{l}\text { At no time-point following exposure to DE was a } \\
\text { significant increase in concentration from baseline } \\
\text { seen for IL-6, TNF-a, S100b, or NSE relative to FA } \\
\text { exposure. Similarly, no significant decrease in BDNF } \\
\text { concentration from baseline was seen following DE } \\
\text { exposure, relative to FA. Furthermore, the repeated } \\
\text { measures ANOVA considered for all time-points and } \\
\text { biomarkers revealed no significant time-exposure } \\
\text { interaction }\end{array}$ & \\
\hline $\begin{array}{l}\text { Oudin et al. } \\
(47)\end{array}$ & $\begin{array}{l}\text { Northern } \\
\text { Sweden }\end{array}$ & Longitudinal & $\begin{array}{l}1806 \text { participants } \\
\text { from the Betula } \\
\text { study }\end{array}$ & $\begin{array}{l}\text { Long-term exposure } \\
\text { to ambient (traffic- } \\
\text { related) air pollution }\end{array}$ & Dementia incidence & $\begin{array}{l}\text { Participants with the highest exposure were more } \\
\text { likely than those with the lowest exposure to be } \\
\text { diagnosed with dementia (Alzheimer's disease or } \\
\text { vascular dementia), with a hazard ratio (HR) of } 1.43 \\
\text { ( } 95 \% \text { Cl: } 0.998-2.05 \text { for the highest vs. the lowest } \\
\text { quartile). The estimates were similar for Alzheimer's } \\
\text { disease (HR 1.38) and vascular dementia (HR 1.47). } \\
\text { The HR for dementia associated with the third quartile } \\
\text { vs. the lowest quartile was } 1.48 \text { ( } 95 \% \mathrm{Cl} \text { : } 1.03-2.11 \text { ) }\end{array}$ & $\begin{array}{l}\text { Education, physical activity, } \\
\text { smoking, sex, BMI, waist-hip } \\
\text { ratio (WHR), alcohol, age, and } \\
\text { apolipoprotein E (ApoE4) }\end{array}$ \\
\hline $\begin{array}{l}\text { Tzivian et al. } \\
\text { (38) }\end{array}$ & $\begin{array}{l}\text { Three } \\
\text { adjacent } \\
\text { cities } \\
\text { (Bochum, } \\
\text { Essen and } \\
\text { Mülheim/ } \\
\text { Ruhr) in } \\
\text { the highly } \\
\text { urbanized } \\
\text { German Ruhr } \\
\text { Area }\end{array}$ & Cross-sectional & $\begin{array}{l}4814 \text { participants } \\
\text { aged } 45-75 \text { years } \\
\text { from a population- } \\
\text { based cohort } \\
\text { study }\end{array}$ & $\begin{array}{l}\text { Long-term air } \\
\text { pollution and traffic } \\
\text { noise exposures }\end{array}$ & $\begin{array}{l}\text { Mild cognitive impairment } \\
\text { in older adults }\end{array}$ & $\begin{array}{l}\text { Long-term exposures to air pollution and traffic noise } \\
\text { were positively associated with } \mathrm{MCl} \text {, mainly with } \\
\text { amnestic subtype. An interquartile range increase } \\
\text { in } \mathrm{PM}_{2.5} \text { and a } 10 \mathrm{~dB}(\mathrm{~A}) \text { increase in } \mathrm{L}_{\mathrm{DEN}} \text { were } \\
\text { associated with overall } \mathrm{MCl}(\mathrm{OR}: 1.16,95 \% \mathrm{Cl} \text { : } \\
1.05-1.27) \text { and }(1.40,95 \% \mathrm{Cl}: 1.0-1.91) \text {, respectively, } \\
\text { and with aMCl }(1.22,96 \% \mathrm{Cl}: 1.08-1.38) \text { and (1.53, } \\
95 \% \mathrm{Cl}: 1.05-2.24) \text {, respectively. In two-exposure } \\
\text { models, AP and noise associations were attenuated } \\
\text { [i.g., for aMCl: } \mathrm{PM}_{2.5} 1.13(0.98,1.30) \text { and LDEN } 1.46 \\
(1.11,1.92)]\end{array}$ & $\begin{array}{l}\text { Age, sex, socioeconomic status, } \\
\text { alcohol consumption in drinks } \\
\text { per week, smoking status, } \\
\text { environmental tobacco smoke, } \\
\text { any regular physical activity, t, BMl, } \\
\text { history of coronary heart disease, } \\
\text { low-density lipoprotein, type } 2 \\
\text { diabetes mellitus, use of statins } \\
\text { and anti-hypertensive medication } \\
\text { during } 7 \text { days before examination } \\
\text { and symptoms of depression }\end{array}$ \\
\hline
\end{tabular}




\begin{tabular}{|c|c|c|c|c|c|c|c|}
\hline Reference & Study location & Study design & Participants & Exposure & Outcomes & Results & Covariates \\
\hline Perera et al. (69) & $\begin{array}{l}\text { New York City, } \\
\text { USA }\end{array}$ & $\begin{array}{l}\text { Prospective } \\
\text { cohort study }\end{array}$ & $\begin{array}{l}183 \text { children } \\
3 \text { years of age }\end{array}$ & $\begin{array}{l}\text { Prenatal } \\
\text { exposure to } \\
\text { PAHs (individual } \\
\text { monitoring at the } \\
\text { 3rd trimester) }\end{array}$ & $\begin{array}{l}\text { Neurodevelopment } \\
\text { in first } 3 \text { years of life: } \\
\text { - Bayley Scale } \\
\text { of Infant } \\
\text { Development. } \\
\text { - Child Behavior } \\
\text { Checklist }\end{array}$ & $\begin{array}{l}\text { Mental development index at age 3: }-5.69 \text { ( } 95 \% \\
\mathrm{Cl}:-9.05 \text { to }-2.33) \text {; cognitive developmental delay: } \\
\text { OR }=2.89 \text { ( } 95 \% \text { Cl: } 1.33-6.25) \text { in the highest vs. the } \\
\text { lowest category of PAH exposure }\end{array}$ & $\begin{array}{l}\text { Prenatal exposure to ETS and } \\
\text { chlorpyrifos, and maternal IQ and } \\
\text { education }\end{array}$ \\
\hline $\begin{array}{l}\text { Windham et al. } \\
\text { (76) }\end{array}$ & $\begin{array}{l}\text { San Francisco, } \\
\text { USA }\end{array}$ & Case-control & $\begin{array}{l}284 \text { children with } \\
\text { ASD diagnosed } \\
\text { before } 9 \text { years } \\
\text { and } 657 \text { controls }\end{array}$ & $\begin{array}{l}\text { Hazardous } \\
\text { air pollutants } \\
\text { (HAPs) levels } \\
\text { at census } \\
\text { tract level } \\
\text { estimated by } \\
\text { EPA Gaussian } \\
\text { Dispersion } \\
\text { Models }\end{array}$ & $\begin{array}{l}\text { Autistic spectrum } \\
\text { disorder }\end{array}$ & $\begin{array}{l}\text { Chemical chlorinated solvents } \\
\text { - Methylene chloride: } \mathrm{OR}=1.50(1.06-2.13) \text { third } \\
\text { quartile } \\
\text { - Trichloroethylene: } \mathrm{OR}=1.47(1.03-2.08) \text { forth quartile } \\
\text { - Vinyl chloride: } \mathrm{OR}=1.75(1.25-2.43) \text { forth quartile } \\
\text { Metals } \\
\text { - Cadmium: } \mathrm{OR}=1.54(1.08-2.20) \text { forth quartile } \\
\text { - Mercury: } \mathrm{OR}=1.92(1.36-2.71) \text { forth quartile } \\
\text { - Nickel: } \mathrm{OR}=1.46(1.04-2.06) \text { forth quartile }\end{array}$ & $\begin{array}{l}\text { Maternal age, education, and } \\
\text { child race }\end{array}$ \\
\hline $\begin{array}{l}\text { Calderón- } \\
\text { Garcidueñas et al. } \\
\text { (23) }\end{array}$ & Mexico & $\begin{array}{l}\text { Cross-sectional } \\
\text { Descriptive }\end{array}$ & $\begin{array}{l}73 \text { children aged } \\
10.5 \pm 3.7 \text { and } \\
9.3 \pm 3.7 \text { years in } \\
\text { two locations }\end{array}$ & $\begin{array}{l}\text { High (Mexico } \\
\text { City)/low } \\
\text { (Polotitlan) levels } \\
\text { of air pollution }\end{array}$ & $\begin{array}{l}\text { Cognitive deficit: } \\
\text { WISC-R } \\
\text { Brain abnormalities: } \\
\text { MRI }\end{array}$ & $\begin{array}{l}\text { Highly exposed children had: } \\
\text { - Poorer performance in the digit span }(p=0.014) \text {, } \\
\text { information }(p=0.019) \text {, and arithmetic }(p=0.031) \\
\text { - Higher proportion of prefrontal white matter } \\
\quad \text { hyperintense lesions than low exposed groups. }\end{array}$ & Age, gender \\
\hline Suglia et al. (58) & Boston, MA & $\begin{array}{l}\text { Prospective } \\
\text { cohort study }\end{array}$ & $\begin{array}{l}202 \text { children } \\
\text { (ages } \\
9.7 \pm 1.7 \text { years) }\end{array}$ & $\begin{array}{l}\text { Black carbon } \\
\text { estimated } \\
\text { by land-use } \\
\text { regression } \\
\text { model }\end{array}$ & $\begin{array}{l}\text { Cognitive functions: } \\
\text { Kaufman Brief } \\
\text { Intelligence Test } \\
\text { (KBIT); The Wide } \\
\text { Range Assessment } \\
\text { of Memory and } \\
\text { Learning (WRAML) }\end{array}$ & $\begin{array}{l}\text { Per interquartile range increasing in log black carbon: } \\
\text { KBIT: matrices score: }-4.0(-7.6 \text { to }-0.5) \text {, composite } \\
\text { score: }-3.4(-6.6 \text { to }-0.3) \text {; WRAML: visual score: }-5.4 \\
(-8.9 \text { to }-1.9) \text {, general score: }-3.9 \text { ( }-7.5 \text { to } 0.3)\end{array}$ & $\begin{array}{l}\text { Age, gender, primary language, } \\
\text { mother's education, passive } \\
\text { smoking, birth weight and blood } \\
\text { lead level. }\end{array}$ \\
\hline Tang et al. (71) & $\begin{array}{l}\text { Chongqing, } \\
\text { China }\end{array}$ & $\begin{array}{l}\text { Prospective } \\
\text { cohort study }\end{array}$ & $\begin{array}{l}110 \text { children } \\
\text { under } 2 \text { years } \\
\text { of age }\end{array}$ & $\begin{array}{l}\text { PAH-DNA } \\
\text { adducts level in } \\
\text { cord blood }\end{array}$ & $\begin{array}{l}\text { Developmental } \\
\text { quotients (DQ) in } \\
\text { motor, adaptive, } \\
\text { language and social } \\
\text { areas at age of } \\
2 \text { years old }\end{array}$ & $\begin{array}{l}\text { Per unit of cord adducts increase:Motor area DQ: } \\
-16.01 \text { ( }-31.30,-0.72) \text {; Average DQ: }-14.57(-28.77, \\
-0.38) \text {; OR for motor developmental delay is } 1.91 \text { (1.22, } \\
2.97) \text { for every } 0.1 \text { unit increase in cord adduct }\end{array}$ & $\begin{array}{l}\text { Cord lead level, environmental } \\
\text { tobacco smoke, sex, gestational } \\
\text { age, and maternal education }\end{array}$ \\
\hline
\end{tabular}




\begin{tabular}{|c|c|c|c|c|c|c|c|}
\hline Reference & Study location & Study design & Participants & Exposure & Outcomes & Results & Covariates \\
\hline Wang et al. (61) & Quanzhou, China & Cross-sectional & $\begin{array}{l}861 \text { children ages } \\
8-10 \text { years }\end{array}$ & $\begin{array}{l}\text { Traffic-related air } \\
\text { pollution: low vs. } \\
\text { exposed school } \\
\text { based on } \mathrm{NO}_{2} \\
\text { and } \mathrm{PM}_{10} \text { levels } \\
\text { (ecologically } \\
\text { determined by } \\
\text { air monitors } \\
\text { and passive } \\
\text { samplers) }\end{array}$ & $\begin{array}{l}\text { Neurobehavioral } \\
\text { functions: cognitive, } \\
\text { motor, sensory and } \\
\text { psychomotor. }\end{array}$ & $\begin{array}{l}\text { Compared high to low exposure schools, odds of } \\
\text { lower performance: visual response speed: visual } \\
\text { simple reaction time-preferred hand test }(\mathrm{OR}=1.67 \\
p=0.044) \text {, and the visual simple reaction time-non- } \\
\text { preferred hand test ( } \mathrm{OR}=1.83, p=0.017) ; \text { Speed and } \\
\text { attention, measured by the continuous performance test } \\
(\mathrm{OR}=2.40, p=0.001) \text {; Accuracy and speed, measured } \\
\text { by the digit symbol test }(\mathrm{OR}=1.38, P=0.019) \text {; } \\
\text { Psychomotor stability, measured by the pursuit aiming } \\
\text { test }(\mathrm{OR}=1.61, P<0.001) \text {; and motor coordination, } \\
\text { measured by the sign register test }(\mathrm{OR}=1.94 \\
P<0.001)\end{array}$ & $\begin{array}{l}\text { Age, BMl, educational attainment } \\
\text { of father, birth weight, delivery } \\
\text { method, passive smoking, open } \\
\text { kitchen, familiarity with computer } \\
\text { games, household fuel, breast } \\
\text { feeding }\end{array}$ \\
\hline \multirow{2}{*}{$\begin{array}{l}\text { Calderón- } \\
\text { Garcidueñas et al. } \\
\text { (22) }\end{array}$} & \multirow[t]{2}{*}{ Mexico } & \multirow[t]{2}{*}{ Cross-sectional } & \multirow{2}{*}{$\begin{array}{l}44 \text { adults and } \\
\text { children aged } \\
2-40 \text { years }\end{array}$} & \multirow{2}{*}{$\begin{array}{l}\text { High (Mexico } \\
\text { City)/low } \\
\text { (Polotitlan) levels } \\
\text { of air pollution }\end{array}$} & \multirow{2}{*}{$\begin{array}{l}\text { The University of } \\
\text { Pennsylvania Smell } \\
\text { Identification Test } \\
\text { (UPSIT) score; } \\
\text { Olfactory bulbs (OB) } \\
\text { pathology }\end{array}$} & $\begin{array}{l}\text { MC subjects had significantly lower UPSIT scores: } \\
34.24 \pm 0.42 \text { vs. controls } 35.7 \pm 0.40, p=0.03\end{array}$ & \multirow[t]{2}{*}{$N / A$} \\
\hline & & & & & & $\begin{array}{l}\text { The exposed groups exhibited remarkable } \\
\text { immunoreactivity to A } \beta 42 \text { and } \alpha \text {-synuclein, and OB } \\
\text { endothelial hyperplasia, and neuronal accumulation of } \\
\text { particles }\end{array}$ & \\
\hline Freire et al. (53) & Spain & Cohort & $\begin{array}{l}210 \text { children ages } \\
4-5 \text { years }\end{array}$ & $\begin{array}{l}\mathrm{NO}_{2} \text { exposure } \\
\text { estimated } \\
\text { by land-use } \\
\text { regression } \\
\text { models }\end{array}$ & $\begin{array}{l}\text { Children's motor } \\
\text { and cognitive } \\
\text { abilities measured } \\
\text { by McCarthy Scales } \\
\text { of Children's Abilities } \\
\text { (MSCA) }\end{array}$ & $\begin{array}{l}\text { For children exposed to higher } \mathrm{NO}_{2}(>24.75 \mathrm{mg} / \\
\left.\mathrm{m}^{3}\right) \text { : general cognitive score: decrease of } 4.19 \text { points; } \\
\text { quantitative memory: decreases of } 6.71 \text { points; working } \\
\text { memory: decrease of } 7.37 \text { points; gross motor: decrease } \\
\text { of } 8.61 \text { points }\end{array}$ & $\begin{array}{l}\text { Birth weight, birth length, } \\
\text { gestational age, smoking during } \\
\text { pregnancy, place of residence, } \\
\text { maternal and paternal educational } \\
\text { level, maternal occupational } \\
\text { status at child age of } 4 \text {, maternal } \\
\text { marital status, mother's parity at } \\
\text { childbirth, breastfeeding (weeks), } \\
\text { mother-to-infant attachment score, } \\
\text { and maternal mental health score }\end{array}$ \\
\hline $\begin{array}{l}\text { Kalkbrenner et al. } \\
\text { (77) }\end{array}$ & $\begin{array}{l}\text { North Carolina } \\
\text { and West } \\
\text { Virginia, USA }\end{array}$ & Case-control & $\begin{array}{l}3212 \text { children } \\
\leq 8 \text { years of age }\end{array}$ & $\begin{array}{l}\text { Prenatal } \\
\text { exposure to } \\
\text { census-tract } \\
\text { HAPS level } \\
\text { estimated by } \\
1996 \text { National } \\
\text { Air Toxics } \\
\text { Assessment } \\
\text { annual-average } \\
\text { model }\end{array}$ & $\begin{array}{l}\text { Autism spectrum } \\
\text { disorder at age } 8\end{array}$ & $\begin{array}{l}\text { Comparing 80th percentile vs. 20th percentile of prenatal } \\
\text { exposure: quinoline (OR = 1.4, 95\% Cl: } 1.0-2.2) \text {; Styrene } \\
\text { (OR = 1.8, 95\% Cl: } 1.0-3.1)\end{array}$ & $\begin{array}{l}\text { Race, maternal education, } \\
\text { maternal age, smoking in } \\
\text { pregnancy, marital status, census } \\
\text { tract median household income, } \\
\text { and urbanicity }\end{array}$ \\
\hline $\begin{array}{l}\text { Calderón- } \\
\text { Garcidueñas et al. } \\
\text { (50) }\end{array}$ & Mexico & Cross-sectional & $\begin{array}{l}30 \text { children } \\
\text { with ages } \\
\sim 7.0 \pm 0.7 \text { years }\end{array}$ & $\begin{array}{l}\text { High (Mexico } \\
\text { City)/low } \\
\text { (Polotitlan) levels } \\
\text { of air pollution }\end{array}$ & Cognitive functions & $\begin{array}{l}\text { Highly exposed healthy children had exhibited selective } \\
\text { impairment in IQ subscales tapping on attention, short- } \\
\text { term memory and learning abilities }\end{array}$ & Year, brain histology \\
\hline
\end{tabular}




\begin{tabular}{|c|c|c|c|c|c|c|c|}
\hline Reference & Study location & Study design & Participants & Exposure & Outcomes & Results & Covariates \\
\hline Guxens et al. (64) & Spain & $\begin{array}{l}\text { Prospective } \\
\text { cohort study }\end{array}$ & $\begin{array}{l}\text { 1,889 children } \\
\sim 14 \text { months }\end{array}$ & $\begin{array}{l}\text { Personal passive } \\
\text { samplers and } \\
\text { land regression } \\
\text { models level } \\
\text { of prenatal } \\
\text { exposures } \\
\text { to } \mathrm{NO}_{2} \text { and } \\
\text { benzene }\end{array}$ & $\begin{array}{l}\text { Cognitive functions: } \\
\text { Bayley Scales of } \\
\text { Infant Development }\end{array}$ & $\begin{array}{l}\text { Exposure to NO2 and benzene are inversely associated } \\
\text { with mental development }(-4.13(-7.06,-1.21) \text { and } \\
-4.37(-6.89,-1.86) \text { for NO2 and benzene, respectively) } \\
\text { in the group of low intakes of fruits/vegetables during } \\
\text { pregnancy }\end{array}$ & $\begin{array}{l}\text { Psychologist, child's sex and age, } \\
\text { maternal education, age, alcohol } \\
\text { use, height, pre-pregnancy BMI, } \\
\text { and dietary, etc. }\end{array}$ \\
\hline Siddique et al. (73) & Delhi, India & Cross-sectional & $\begin{array}{l}1819 \text { children } \\
2-17 \text { years }\end{array}$ & $\begin{array}{l}\mathrm{PM}_{10} \text { estimated } \\
\text { by surrounding } \\
\text { monitoring } \\
\text { stations }\end{array}$ & $\mathrm{ADHD}$ & $\begin{array}{l}<120 \mu \mathrm{g} / \mathrm{m} 3: 1.00 \text { (referent); } 120-139 \mu \mathrm{g} / \mathrm{m} 3: 1.824 \\
\text { (1.070-3.629); } 140-200 \mu \mathrm{g} / \mathrm{m} 3: 2.201 \text { (1.162-5.032); } \\
>200 \mu \mathrm{g} / \mathrm{m} 3: 2.770 \text { (1.381-5.555) }\end{array}$ & $\begin{array}{l}\text { Age, gender, socioeconomic } \\
\text { status, and parental smoking, } \\
\text { indoor pollution }\end{array}$ \\
\hline Volk et al. (78) & California, USA & Case-control & $\begin{array}{l}563 \text { children } \\
\text { between } 24 \text { and } \\
60 \text { months }\end{array}$ & $\begin{array}{l}\text { Proximity to } \\
\text { freeways }\end{array}$ & Autism & $\begin{array}{l}\text { Mothers living }>309 \mathrm{~m} \text { from freeways: } \mathrm{OR}=1.86 ; 95 \% \\
\text { Cl: } 1.04-3.45 . \text { Proximity to a freeway during the third } \\
\text { trimester: } \mathrm{OR}=2.22 ; 95 \% \mathrm{Cl}: 1.16-4.42\end{array}$ & $\begin{array}{l}\text { Child sex, child race/ethnicity, } \\
\text { maximum education of parents, } \\
\text { maternal, and maternal smoking } \\
\text { during pregnancy }\end{array}$ \\
\hline Clark et al. (51) & United Kingdom & Cross-sectional & $\begin{array}{l}719 \text { children who } \\
\text { were 9-10 years }\end{array}$ & $\begin{array}{l}\mathrm{SO}_{2} \text { determined } \\
\text { by emission } \\
\text { dispersion and } \\
\text { regression } \\
\text { models }\end{array}$ & $\begin{array}{l}\text { Cognitive functions; } \\
\text { working and } \\
\text { episodic memory }\end{array}$ & $\begin{array}{l}\text { No significant findings for association between } \mathrm{SO}_{2} \\
\text { and cognitive functions measured by the Child Memory } \\
\text { Scale, and The Search and Memory Task }\end{array}$ & $\begin{array}{l}\text { Age, gender, mother's educational } \\
\text { level, parental employment status, } \\
\text { crowding in the home, home } \\
\text { ownership, long-standing illness, } \\
\text { main language spoken at home, } \\
\text { parental support for school work, } \\
\text { and classroom window glazing, } \\
\text { traffic noise }\end{array}$ \\
\hline $\begin{array}{l}\text { van Kempen et al. } \\
\text { (60) }\end{array}$ & Netherlands & Cross-sectional & $\begin{array}{l}553 \text { children (age } \\
\text { 9-11 years) }\end{array}$ & $\begin{array}{l}\text { Yearly average } \\
\text { concentration } \\
\text { of } \mathrm{NO}_{2} \text { and } \\
\mathrm{PM}_{10} \text { estimated } \\
\text { by land-use } \\
\text { regression } \\
\text { model }\end{array}$ & $\begin{array}{l}\text { Cognitive } \\
\text { performance using } \\
\text { the Neurobehavioral } \\
\text { Evaluation System } \\
\text { (NES) }\end{array}$ & $\begin{array}{l}\text { Air pollution at school was found to be associated with } \\
\text { a decrease in the span length measured during Digit } \\
\text { Memory Span Test }(-0.16(-0.28,-0.04) \text { for each } \\
\left.10 \mathrm{mg} / \mathrm{m}^{3} \text { increase in } \mathrm{NO}_{2}\right)\end{array}$ & $\begin{array}{l}\text { Age, gender, employment status, } \\
\text { crowding, home ownership, } \\
\text { mother's education, long-standing } \\
\text { illness, main language spoken at } \\
\text { home, parental support, and type } \\
\text { of window glazing at school }\end{array}$ \\
\hline Becerra et al. (79) & $\begin{array}{l}\text { Los Angeles, } \\
\text { California }\end{array}$ & Case-control & $\begin{array}{l}7,594 \text { cases and } \\
75,635 \text { controls } \\
3-14 \text { years of age }\end{array}$ & $\begin{array}{l}\mathrm{CO} . \mathrm{NO}_{2}, \mathrm{NO} \\
\mathrm{O}_{3}, \mathrm{PM}_{2.5}, \mathrm{PM} 10 \\
\text { from nearest } \\
\text { monitoring } \\
\text { stations and } \\
\text { LUR models }\end{array}$ & $\begin{array}{l}\text { Autistic disorders } \\
\text { diagnosed between } \\
36 \text { and } 71 \text { months } \\
\text { of age }\end{array}$ & $\begin{array}{l}\text { For an IQR increase, there was } 12-15 \% \text { increase in odds } \\
\text { of autism for } \mathrm{O}_{3} \text { and } \mathrm{PM}_{2.5} \text {; and } 3-9 \% \text { relative increases } \\
\text { in odds for } \mathrm{LUR} \text {-based } \mathrm{NO} \text { and } \mathrm{NO}_{2} \text { exposure estimates. } \\
\text { Associations were stronger among children of mothers } \\
\text { <high school education }\end{array}$ & $\begin{array}{l}\text { Maternal age, maternal place of } \\
\text { birth, race/ethnicity, education, } \\
\text { type of birth, parity, insurance } \\
\text { type, and gestational age }\end{array}$ \\
\hline Jung et al. (45) & Taiwan & Cohort & $\begin{array}{l}49,073 \text { children } \\
\text { age less than } \\
3 \text { years }\end{array}$ & $\begin{array}{l}\text { Exposures to } \\
\mathrm{O}_{3}, \mathrm{CO}, \mathrm{NO} 2 \\
\mathrm{SO}_{2}, \mathrm{PM}_{10} \\
\text { during preceding } \\
1-4 \text { years } \\
\text { estimated using } \\
\text { inverse distance } \\
\text { weighting }\end{array}$ & $\begin{array}{l}\text { Autism spectrum } \\
\text { disorder }\end{array}$ & $\begin{array}{l}\text { Previous year exposure was associated with: } 59 \% \text { risk } \\
\text { increase per } 10 \mathrm{ppb} \text { increase in } \mathrm{O}_{3} ; 37 \% \text { risk increase } \\
\text { per } 10 \mathrm{ppb} \text { in } \mathrm{CO} ; 340 \% \text { risk increase per } 10 \mathrm{ppb} \\
\text { increase in } \mathrm{NO}_{2} ; 17 \% \text { risk increase per } 1 \mathrm{ppb} \text { in } \mathrm{SO}_{2}\end{array}$ & $\begin{array}{l}\text { Gender, municipal-level } \\
\text { socioeconomic status (SES) of } \\
\text { parents, and comorbidities }\end{array}$ \\
\hline
\end{tabular}




\begin{tabular}{|c|c|c|c|c|c|c|c|}
\hline Reference & Study location & Study design & Participants & Exposure & Outcomes & Results & Covariates \\
\hline Newman et al. (74) & $\begin{array}{l}\text { Cincinnati, Ohio, } \\
\text { USA }\end{array}$ & Cohort & $\begin{array}{l}576 \text { children ages } \\
6.9 \pm 0.3 \text { years }\end{array}$ & $\begin{array}{l}\text { Elemental } \\
\text { carbon } \\
\text { attributed to } \\
\text { traffic (ECAT) } \\
\text { during first year } \\
\text { of life estimated } \\
\text { by LUR }\end{array}$ & $\begin{array}{l}\text { ADHD symptoms } \\
\text { at } 7 \text { years of age } \\
\text { assessed by } \\
\text { the Behavioral } \\
\text { Assessment System } \\
\text { for Children, 2nd } \\
\text { Edition }\end{array}$ & $\begin{array}{l}\text { Highest tertile of ECAT during the child's first year of life } \\
\text { was significantly associated with Hyperactivity } T \text {-scores } \\
\text { in the "at risk" range at } 7 \text { years of age (adjusted odds } \\
\text { ratio (aOR) = 1.7; 95\% Cl: } 1.0-2.7) \text {. Stronger association } \\
\text { in children whose mothers had higher education }\end{array}$ & $\begin{array}{l}\text { Environmental tobacco smoke } \\
\text { during pregnancy and first year of } \\
\text { life, age of home, Maternal and } \\
\text { paternal education, household } \\
\text { income, insurance status race/ } \\
\text { ethnicity, duration of breastfeeding, } \\
\text { and childcare attendance }\end{array}$ \\
\hline Roberts et al. (81) & 14 U.S. states & Case-control & $\begin{array}{l}325 \text { cases, } \\
22,101 \text { controls } \\
\text { ages }\end{array}$ & $\begin{array}{l}\text { U.S. } \\
\text { Environmental } \\
\text { Protection } \\
\text { Agency- } \\
\text { modeled levels } \\
\text { of hazardous air } \\
\text { pollutants at the } \\
\text { time and place } \\
\text { of birth }\end{array}$ & $\begin{array}{l}\text { Autism spectrum } \\
\text { disorder diagnosis } \\
\text { by telephone } \\
\text { administration of the } \\
\text { Autism Diagnostic } \\
\text { Interview-Revised }\end{array}$ & $\begin{array}{l}\text { Perinatal exposures to the highest vs. lowest quintile of } \\
\text { diesel, lead, manganese, and cadmium, and an overall } \\
\text { measure of metals were significantly associated with } \\
\text { ASD with odds ratios ranging from } 1.5 \text { (for cadmium) to } \\
2.0 \text { (for diesel). Associations were stronger for boys than } \\
\text { for girls }\end{array}$ & $\begin{array}{l}\text { Census tract median income } \\
\text { and percent of residents with } \\
\text { a college education, mother's } \\
\text { parents' education, index child's } \\
\text { current family income, educational } \\
\text { attainment of the mother's partner, } \\
\text { smoking, year of birth, maternal } \\
\text { age at birth, and air pollution } \\
\text { prediction model year }\end{array}$ \\
\hline Volk et al. (78) & California, USA & Case-control & $\begin{array}{l}279 \text { cases and } \\
245 \text { controls } \\
\text { ages of } 24 \text { and } \\
60 \text { months }\end{array}$ & $\begin{array}{l}\text { Traffic-related } \\
\text { pollution } \\
\text { exposure for } \\
\text { each trimester } \\
\text { of pregnancy } \\
\text { and first year of } \\
\text { life estimated } \\
\text { by dispersion } \\
\text { models and } \\
\text { inverse distance } \\
\text { weighting }\end{array}$ & $\begin{array}{l}\text { Autism diagnosed } \\
\text { by Autism } \\
\text { Diagnostic } \\
\text { Observation } \\
\text { Schedules (ADOS) } \\
\text { and Autism } \\
\text { Diagnostic Interview- } \\
\text { Revised (ADI-R) }\end{array}$ & $\begin{array}{l}\text { Children with highest levels of modeled TRP during } \\
\text { first year of life, and all trimesters of pregnancy were } \\
\text { significantly more likely to have autism compared to } \\
\text { children with the lowest exposure. Individual pollutants } \\
\text { including } \mathrm{PM}_{2.5}, \mathrm{PM}_{10} \text {, and } \mathrm{NO}_{2} \text { were also significantly } \\
\text { associated at all windows }\end{array}$ & $\begin{array}{l}\text { Gender, ethnicity, maximum } \\
\text { education level of the parents, } \\
\text { maternal age, maternal smoking } \\
\text { during pregnancy, population } \\
\text { density }\end{array}$ \\
\hline Gong et al. (75) & $\begin{array}{l}\text { Stockholm, } \\
\text { Sweden }\end{array}$ & Cohort & $\begin{array}{l}3,426 \text { twins up to } \\
12 \text { years of age }\end{array}$ & $\begin{array}{l}\text { Residence } \\
\text { time-weighted } \\
\text { concentration } \\
\text { of } \mathrm{PM}_{10} \text { and } \\
\mathrm{NO}_{\times} \text {during } \\
\text { pregnancy, the } \\
\text { first year, and } \\
\text { the ninth year } \\
\text { of life using } \\
\text { dispersion } \\
\text { modeling }\end{array}$ & $\begin{array}{l}\text { Autism spectrum } \\
\text { disorders (ASD) } \\
\text { and attention- } \\
\text { deficit hyperactivity } \\
\text { disorder (ADHD) } \\
\text { at } 9 \text { or } 12 \text { years } \\
\text { old using DSM-IV } \\
\text { criteria }\end{array}$ & $\begin{array}{l}\text { No clear or consistent associations were found between } \\
\text { air pollution exposure during any of the three time } \\
\text { windows and any of the neurodevelopmental outcomes. }\end{array}$ & $\begin{array}{l}\text { Gender, parity, gestational age, } \\
\text { birthweight, maternal age at } \\
\text { birth, maternal smoking during } \\
\text { pregnancy, maternal marital } \\
\text { status, parental education, } \\
\text { family disposable income during } \\
\text { pregnancy, child's first year of } \\
\text { life, and the ninth year of life, and } \\
\text { neighborhood deprivation index }\end{array}$ \\
\hline
\end{tabular}




\begin{tabular}{|c|c|c|c|c|c|c|c|}
\hline Reference & Study location & Study design & Participants & Exposure & Outcomes & Results & Covariates \\
\hline Guxens et al. (65) & $\begin{array}{l}\text { Six European } \\
\text { countries }\end{array}$ & Cohort & $\begin{array}{l}9482 \text { children } \\
\text { aged } 1-6 \text { years }\end{array}$ & $\begin{array}{l}\mathrm{NO}_{2}, \mathrm{NO}_{x}, \mathrm{PM}_{2.5} \\
\text { absorbance, } \\
\mathrm{PM}_{10}, \mathrm{PM}_{2.5}, \\
\mathrm{PM}_{\text {coarse }} \\
\text { concentrations } \\
\text { birth home } \\
\text { addresses } \\
\text { were estimated } \\
\text { by land-use } \\
\text { regression } \\
\text { models }\end{array}$ & $\begin{array}{l}\text { Cognitive and } \\
\text { motor development } \\
\text { evaluated by parents } \\
\text { or psychologist }\end{array}$ & $\begin{array}{l}\mathrm{NO}_{2} \text { was associated with reduced psychomotor } \\
\text { development (global psychomotor development score } \\
\text { decreased by } 0.68 \text { points }(95 \% \mathrm{Cl}:-1.25 \text { to }-0.11 \text { ) per } \\
\left.\text { increase of } 10 \mu \mathrm{g} / \mathrm{m}^{3}\right)\end{array}$ & $\begin{array}{l}\text { Maternal age at delivery, } \\
\text { educational level, country of } \\
\text { birth, smoking during pregnancy, } \\
\text { parity, maternal height and pre- } \\
\text { pregnancy weight, child's sex and } \\
\text { date of birth }\end{array}$ \\
\hline Kim et al. (67) & South Korea & Cohort & $\begin{array}{l}520 \text { mother-child } \\
\text { pairs (children } \\
\leq 24 \text { months). }\end{array}$ & $\begin{array}{l}\mathrm{PM}_{10} \text { and } \mathrm{NO}_{2} \\
\text { during entire } \\
\text { pregnancy } \\
\text { estimated by } \\
\text { inverse distance } \\
\text { weighting }\end{array}$ & $\begin{array}{l}\text { Neurodevelopment } \\
\text { during the first } \\
24 \text { months, } \\
\text { assessed by Korean } \\
\text { Bayley Scale of } \\
\text { Infant Development } \\
\text { II (K-BSID-II) }\end{array}$ & $\begin{array}{l}\text { There were negative associations between maternal } \\
\text { exposure to } \mathrm{PM}_{10} \text { and mental developmental index } \\
(\beta=-2.83 ; p=0.003) \text { and psychomotor developmental } \\
\text { index ( } \beta=-3.00 ; p=0.002) \text {. } \mathrm{NO}_{2} \text { exposure was } \\
\text { related with impairment of psychomotor development } \\
(\beta=-1.30 ; p=0.05)\end{array}$ & $\begin{array}{l}\text { Maternal age, maternal education, } \\
\text { family income, birthweight, sex, } \\
\text { and gestational age }\end{array}$ \\
\hline Lin et al. (57) & Taiwan & Cohort & $\begin{array}{l}533 \text { mother-infant } \\
\text { pairs (children } \\
\leq 18 \text { months) }\end{array}$ & $\begin{array}{l}\mathrm{PM}_{10}, \mathrm{CO}, \mathrm{SO}_{2} \\
\mathrm{NO}_{2}, \mathrm{O}_{3} \text { and } \\
\text { hydrocarbons } \\
\text { measured } \\
\text { at air quality } \\
\text { monitoring } \\
\text { stations }\end{array}$ & $\begin{array}{l}\text { Neurobehavioral } \\
\text { development when } \\
\text { the children were } \\
\text { 0-18 months } \\
\text { reported by parents }\end{array}$ & $\begin{array}{l}\text { Exposures to } \mathrm{SO}_{2} \text { during first trimester }(\beta=-0.083 \text {, } \\
\mathrm{SE}=0.030), 2 \text { nd and } 3 \text { rd trimester }(\beta=-0.114 \\
\mathrm{SE}=0.045), \text { and birth } 12 \text { months }(\beta=-0.091 \text {, } \\
\mathrm{SE}=0.034) \text { were associated with decreased fine motor } \\
\text { scores. Non-methane hydrocarbon during } 2^{\text {nd }} \text { and } 3^{\text {rd }} \\
\text { trimester was also associated with decreased gross } \\
\text { motor: }(\beta=-8.742, \mathrm{SE}=3.512)\end{array}$ & $\begin{array}{l}\text { Maternal education, maternal } \\
\text { education, environmental tobacco } \\
\text { smoke exposure, infant gestational } \\
\text { age, breastfeeding, and parental } \\
\text { nursery type }\end{array}$ \\
\hline Lovasi et al. (68) & New York, USA & Cohort & $\begin{array}{l}326 \text { mother-infant } \\
\text { pairs }\end{array}$ & $\begin{array}{l}\text { Polycyclic } \\
\text { aromatic } \\
\text { hydrocarbon } \\
\text { (PAH) measured } \\
\text { by personal } \\
\text { monitoring at } \\
\text { a single point } \\
\text { during third } \\
\text { trimester }\end{array}$ & $\begin{array}{l}\text { Cognitive scores at } \\
\text { age } 5 \text { based on the } \\
\text { Wechsler Preschool } \\
\text { and Primary Scale of } \\
\text { Intelligence-Revised } \\
\text { (WPPSI-R) }\end{array}$ & $\begin{array}{l}\text { Prenatal PAH exposure above the median predicted } \\
\text { 3.5-point lower total WPPSI-R scores and 3.9-point } \\
\text { lower verbal scores }\end{array}$ & $\begin{array}{l}\text { Sex, ethnicity, maternal education, } \\
\text { maternal IQ score, environmental } \\
\text { tobacco smoke exposure in the } \\
\text { home, quality of the caretaking } \\
\text { environment, and household } \\
\text { English language exposure. }\end{array}$ \\
\hline Cowell et al. (63) & $\begin{array}{l}\text { Boston, } \\
\text { Massachusetts, } \\
\text { USA }\end{array}$ & Cohort & $\begin{array}{l}258 \text { mother-child } \\
\text { dyads }\end{array}$ & $\begin{array}{l}\text { Black carbon } \\
\text { estimated using } \\
\text { LUR }\end{array}$ & $\begin{array}{l}\text { Cognitive scores } \\
\text { measured by Wide } \\
\text { Range Assessment } \\
\text { of Memory and } \\
\text { Learning (WRAML2) } \\
\text { at age } 6\end{array}$ & $\begin{array}{l}\text { The main effect for black carbon was not significant } \\
\text { for any WRAML2 index; however, in stratified analyses, } \\
\text { among boys with high exposure to prenatal stress, } \\
\text { Attention Concentration Index scores were on average } \\
9.5 \text { points lower for those with high compared to low } \\
\text { prenatal black carbon exposure }\end{array}$ & $\begin{array}{l}\text { Maternal age, race/ethnicity, } \\
\text { education, smoking status, and } \\
\text { birthweight }\end{array}$ \\
\hline
\end{tabular}




\begin{tabular}{|c|c|c|c|c|c|c|c|}
\hline Reference & Study location & Study design & Participants & Exposure & Outcomes & Results & Covariates \\
\hline Harris et al. (55) & $\begin{array}{l}\text { Massachusetts, } \\
\text { USA }\end{array}$ & Cohort & $\begin{array}{l}1,109 \text { mother- } \\
\text { child pairs }\end{array}$ & $\begin{array}{l}\text { Black carbon } \\
(\mathrm{BC}) \text { and } \mathrm{PM}_{2.5} \\
\text { exposures } \\
\text { (LUR), and } \\
\text { residential } \\
\text { proximity to } \\
\text { major roadways, } \\
\text { and near- } \\
\text { residence traffic } \\
\text { density during } \\
\text { late pregnancy } \\
\text { and childhood }\end{array}$ & $\begin{array}{l}\text { Verbal and non- } \\
\text { verbal intelligence, } \\
\text { visual motor abilities, } \\
\text { and visual memory } \\
\text { at age } 8\end{array}$ & $\begin{array}{l}\text { Compared with children living } \geq 200 \mathrm{~m} \text { from a major } \\
\text { roadway at birth, those living }<50 \mathrm{~m} \text { away had lower } \\
\text { non-verbal IQ }(-7.5 \text { points; } 95 \% \mathrm{Cl}:-13.1,-1.9) \text {, and } \\
\text { somewhat lower verbal IQ (-3.8 points; } 95 \% \mathrm{Cl}-8.2 \\
\text { to } 0.6 \text { ) and visual motor abilities ( }-5.3 \text { points; } 95 \% \mathrm{Cl} \text { : } \\
-11.0 \text { to } 0.4 \text { ). }\end{array}$ & $\begin{array}{l}\text { Sex, age at cognitive testing, } \\
\text { breastfeeding duration, maternal } \\
\text { IQ, parity, age at enrollment, } \\
\text { marital status, education, race/ } \\
\text { ethnicity, smoking status, second } \\
\text { hand smoke during pregnancy, } \\
\text { father education, household } \\
\text { ownership of gas stove, annual } \\
\text { income, and neighborhood } \\
\text { income }\end{array}$ \\
\hline $\begin{array}{l}\text { Jedrychowski } \\
\text { et al. (66) }\end{array}$ & Krakow, Poland & Cohort & 170 children & $\begin{array}{l}\text { Polycyclic } \\
\text { aromatic } \\
\text { hydrocarbons } \\
\text { (PAHs) } \\
\text { exposures } \\
\text { indicated by } \\
\text { PAH-DNA } \\
\text { adducts in cord } \\
\text { blood }\end{array}$ & $\begin{array}{l}\text { Cognitive function } \\
\text { at age } 7 \text { assessed } \\
\text { by at the Wechsler } \\
\text { Intelligence Scale } \\
\text { for Children-Revised } \\
\text { (WISC-R) }\end{array}$ & $\begin{array}{l}\text { Relative risk of depressed verbal IQ index increased } \\
\text { threefold with an In-unit increase in cord blood adducts } \\
\text { (relative risk (RR) }=3.0,95 \% \mathrm{Cl} \text { : 1.3-6.8) }\end{array}$ & $\begin{array}{l}\text { Postnatal indoor PAH exposure, } \\
\text { maternal education, child's gender, } \\
\text { parity, breastfeeding practice, and } \\
\text { prenatal and postnatal ETS }\end{array}$ \\
\hline Kicinski et al.(56) & Belgium & Cohort & $\begin{array}{l}606 \text { adolescents } \\
\text { aged } \\
14.9 \pm 0.7 \text { years }\end{array}$ & $\begin{array}{l}\text { Biomarker of } \\
\text { benzene and } \\
\text { the amount of } \\
\text { contact with } \\
\text { traffic using } \\
\text { inverse distance } \\
\text { weighting }\end{array}$ & $\begin{array}{l}\text { Neurobehavioral } \\
\text { function assessed } \\
\text { using the } \\
\text { Neurobehavioral } \\
\text { Evaluation System } \\
\text { computerized } \\
\text { battery of tests } \\
\text { (NES3) }\end{array}$ & $\begin{array}{l}\text { One SD increase in traffic exposure was associated with } \\
\text { a } 0.26 \mathrm{SD} \text { decrease in sustained attention ( } 95 \% \text { credible } \\
\text { interval: }-0.02 \text { to }-0.51)\end{array}$ & $\begin{array}{l}\text { Gender, age, smoking, passive } \\
\text { smoking, level of education of the } \\
\text { mother, socioeconomic status, } \\
\text { time of the day, and day of the } \\
\text { week }\end{array}$ \\
\hline $\begin{array}{l}\text { Kalkbrenner et al. } \\
\text { (83) }\end{array}$ & USA & Case-control & $\begin{array}{l}979 \text { cases and } \\
14,666 \text { controls }\end{array}$ & $\mathrm{PM}_{10}$ & $\begin{array}{l}\text { Autism spectrum } \\
\text { disorder (ASD) }\end{array}$ & $\begin{array}{l}\text { PM10 exposure during the third trimester was associated } \\
\text { with } \mathrm{ASD}(\mathrm{OR}=1.36,95 \% \mathrm{Cl}: 1.13-1.63)\end{array}$ & $\begin{array}{l}\text { Race, maternal education, } \\
\text { maternal age, smoking in } \\
\text { pregnancy, marital status, census } \\
\text { tract median household income, } \\
\text { and urbanicity }\end{array}$ \\
\hline Raz et al. (84) & USA & $\begin{array}{l}\text { Nested } \\
\text { case-control }\end{array}$ & $\begin{array}{l}245 \text { cases and } \\
1,522 \text { randomly } \\
\text { selected controls }\end{array}$ & $\begin{array}{l}\mathrm{PM}_{10-2.5} \\
\text { and } \mathrm{PM}_{2.5} \\
\text { concentration } \\
\text { before, during, } \\
\text { and after } \\
\text { pregnancy } \\
\text { estimated } \\
\text { by validated } \\
\text { spatiotemporal } \\
\text { models }\end{array}$ & $\begin{array}{l}\text { Autism spectrum } \\
\text { disorder (ASD) } \\
\text { assessed by } \\
\text { parental report } \\
\text { (validated) }\end{array}$ & $\begin{array}{l}\mathrm{PM}_{2.5} \text { exposure during pregnancy was associated } \\
\text { with increased odds of } \mathrm{ASD} \text {; with an adjusted odds } \\
\text { ratio (OR) for ASD per interquartile range (IQR) higher } \\
\mathrm{PM}_{2.5}\left(4.42 \mu \mathrm{g} / \mathrm{m}^{3}\right) \text { of } 1.57 \text { ( }(95 \% \mathrm{Cl}: 1.22-2.03) \text {. The } \\
\text { association between } \mathrm{ASD} \text { and } \mathrm{PM}_{2.5} \text { was stronger for } \\
\text { exposure during the third trimester }\end{array}$ & $\begin{array}{l}\text { Child's birth year, birth month, and } \\
\text { sex, maternal age at birth, paternal } \\
\text { age at birth, and median census } \\
\text { tract income in the birth year }\end{array}$ \\
\hline
\end{tabular}




\begin{tabular}{|c|c|c|c|c|c|c|c|}
\hline Reference & Study location & Study design & Participants & Exposure & Outcomes & Results & Covariates \\
\hline Sunyer et al. (59) & Catalonia, Spain & Cohort & $\begin{array}{l}2,715 \text { children } \\
\text { aged } 7-10 \text { years }\end{array}$ & $\begin{array}{l}\text { Elemental } \\
\text { carbon (EC), } \\
\mathrm{NO}_{2} \text {, and } \\
\text { ultrafine particle } \\
\text { number (UFP) } \\
\text { measured by air } \\
\text { monitors set at } \\
\text { schools }\end{array}$ & $\begin{array}{l}\text { Cognitive } \\
\text { development } \\
\text { assessed with the } \\
\text { n-back and the } \\
\text { attentional network } \\
\text { tests }\end{array}$ & $\begin{array}{l}\text { Children attending schools with higher levels of EC, } \\
\mathrm{NO}_{2} \text {, and UFP both indoors and outdoors experienced } \\
\text { substantially smaller growth in all the cognitive } \\
\text { measurements }\end{array}$ & $\begin{array}{l}\text { Age, sex, maternal education, } \\
\text { socioeconomic status, and air } \\
\text { pollution exposure at home }\end{array}$ \\
\hline Talbott et al. (85) & $\begin{array}{l}\text { Pennsylvania, } \\
\text { USA }\end{array}$ & Case-control & $\begin{array}{l}217 \text { cases and } \\
226 \text { controls }\end{array}$ & $\begin{array}{l}\mathrm{PM}_{2.5} \text { predicted } \\
\text { by LUR during } \\
\text { pre-pregnancy, } \\
\text { trimesters one } \\
\text { through three, } \\
\text { pregnancy, } \\
\text { years one and } \\
\text { two of life and } \\
\text { cumulative } \\
\text { (starting from } \\
\text { pre-pregnancy) }\end{array}$ & $\begin{array}{l}\text { Causes of } \\
\text { autism spectrum } \\
\text { disorder (ASD) } \\
\text { assessed by Social } \\
\text { Communication } \\
\text { Questionnaire } \\
\text { (SCQ) or medical } \\
\text { documentation. }\end{array}$ & $\begin{array}{l}\text { An IQR increased in average exposure to } \mathrm{PM}_{2.5}(\mu \mathrm{g} / \\
\left.\left.\mathrm{m}^{3}\right) \text { during year } 2 \text { postnatal (aOR: } 1.45(1.01-2.08)\right) \text {, } \\
\text { and during pre-pregnancy through year } 2(\mathrm{aOR}: 1.51 \\
(1.01-2.26)) \text { were associated with ASD. }\end{array}$ & $\begin{array}{l}\text { Mother's age, education, race, } \\
\text { and smoking. }\end{array}$ \\
\hline Talbott et al. (86) & $\begin{array}{l}\text { Pennsylvania, } \\
\text { USA }\end{array}$ & Case-control & $\begin{array}{l}217 \text { ASD cases } \\
\text { and } 5231 \\
\text { controls }\end{array}$ & $\begin{array}{l}30 \\
\text { neurotoxicants } \\
\text { assessed by US } \\
\text { EPA National } \\
\text { Air Toxics } \\
\text { Assessment }\end{array}$ & $\begin{array}{l}\text { Causes of } \\
\text { autism spectrum } \\
\text { disorder (ASD) } \\
\text { assessed by Social } \\
\text { Communication } \\
\text { Questionnaire } \\
\text { (SCQ) or medical } \\
\text { documentation }\end{array}$ & $\begin{array}{l}\text { Comparing fourth to first quartile exposures for all births, } \\
\text { the adjusted OR for styrene was } 1.61(95 \% \mathrm{Cl}=1.08- \\
2.40, p=0.018) \text {. Chromium also exhibited an elevated } \\
\text { OR of } 1.60(95 \% \mathrm{Cl}=1.08-2.38, p=0.020)\end{array}$ & $\begin{array}{l}\text { Mother's age, race, education, } \\
\text { smoking, child's birth year, and } \\
\text { child's sex }\end{array}$ \\
\hline $\begin{array}{l}\text { Calderón- } \\
\text { Garcidueñas et al. } \\
\text { (49) }\end{array}$ & $\begin{array}{l}\text { Mexico City } \\
\text { Metropolitan } \\
\text { Area (MCMA) } \\
\text { and small } \\
\text { cites in Mexico } \\
\text { (Zacatlán and } \\
\text { Huachinango, } \\
\text { Puebla; } \\
\text { Zitácuaro, } \\
\text { Michoacán; } \\
\text { Puerto } \\
\text { Escondido, } \\
\text { Oaxaca) }\end{array}$ & Case-control & $\begin{array}{l}\text { Children cohort } \\
\text { from MC and } \\
\text { control location } \\
\text { consisting of } \\
\text { small cities in } \\
\text { Mexico }\end{array}$ & $\begin{array}{l}\text { Exposure to high } \\
\text { concentrations } \\
\text { of air pollutants, } \\
\text { i.e., } \mathrm{PM}_{2.5} \text { and } \\
\text { ozone }\end{array}$ & $\begin{array}{l}\text { Impact on } \\
\text { neurovascular unit } \\
\text { dysfunction and } \\
\text { risk of Alzheimer's } \\
\text { disease }\end{array}$ & $\begin{array}{l}\text { Major findings in MC residents included leaking } \\
\text { capillaries and small arterioles with extravascular lipids } \\
\text { and erythrocytes, lipofuscin in pericytes, smooth muscle } \\
\text { and EC, thickening of cerebrovascular basement } \\
\text { membranes with small deposits of amyloid, patchy } \\
\text { absence of the perivascular glial sheet, enlarged } \\
\text { Virchow-Robin spaces and nanosize particles } \\
(20-48 \mathrm{~nm}) \text { in endothelial cells, basement membranes, } \\
\text { axons and dendrites. The integrity of the neurovascular } \\
\text { unit, an interactive network of vascular, glial and neuronal } \\
\text { cells is compromised in MC young residents }\end{array}$ & \\
\hline
\end{tabular}




\begin{tabular}{|c|c|c|c|c|c|c|c|}
\hline Reference & Study location & Study design & Participants & Exposure & Outcomes & Results & Covariates \\
\hline Chiu et al. (62) & Boston, MA, USA & Cohort & $\begin{array}{l}267 \text { singleton } \\
\text { full-term urban } \\
\text { children from } \\
\text { ACCESS project }\end{array}$ & $\begin{array}{l}\text { Exposure } \\
\text { to prenatal } \\
\text { particulate air } \\
\text { pollution }\end{array}$ & $\begin{array}{l}\text { Neurodevelopment } \\
\text { in urban children }\end{array}$ & $\begin{array}{l}\text { The study suggests the associations between } \\
\text { higher } \mathrm{PM}_{2.5} \text { levels at } 31-38 \text { weeks with lower IQ, } \\
\text { at } 20-26 \text { weeks gestation with increased OEs, at } \\
32-36 \text { weeks with slower HRT, and at } 22-40 \text { weeks } \\
\text { with increased HRT-SE among boys, while significant } \\
\text { associations were found in memory domains in girls } \\
\text { (higher } \mathrm{PM}_{2.5} \text { exposure at } 18-26 \text { weeks with reduced } \\
\text { VIM, at } 12-20 \text { weeks with reduced GM). Increased } \\
\mathrm{PM}_{2.5} \text { exposure in specific prenatal windows may be } \\
\text { associated with poorer function across memory and } \\
\text { attention domains with variable associations based on } \\
\text { sex }\end{array}$ & $\begin{array}{l}\text { Maternal age, race, and } \\
\text { educational status, child's sex, } \\
\text { date of birth, parity, gestational } \\
\text { age at birth, and birth weight, } \\
\text { smoking status, breast feeding } \\
\text { duration, and children's blood lead } \\
\text { levels }\end{array}$ \\
\hline Forns et al. (52) & $\begin{array}{l}\text { Barcelona } \\
\text { (Catalonia, Spain) }\end{array}$ & Cross-sectional & $\begin{array}{l}2897 \text { children } \\
7-11 \text { years of age } \\
\text { from } 39 \text { schools } \\
\text { in Barcelona, } \\
\text { during 2012-13 }\end{array}$ & $\begin{array}{l}\text { Exposure to } \\
\text { traffic-related } \\
\text { air pollution and } \\
\text { noise at school }\end{array}$ & $\begin{array}{l}\text { Behavioral problems } \\
\text { in school-age } \\
\text { children }\end{array}$ & $\begin{array}{l}\text { Exposure to TRAPs at school was associated with } \\
\text { increased behavioral problems in schoolchildren. Noise } \\
\text { exposure at school was associated with more ADHD } \\
\text { symptoms. Interquartile range increases in indoor and } \\
\text { outdoor EC, BC, and NO2 concentrations were positively } \\
\text { associated with SDQ total difficulties scores (suggesting } \\
\text { more frequent behavioral problems) in adjusted } \\
\text { multivariate models, whereas noise was significantly } \\
\text { associated with ADHD-DSM-IV scores }\end{array}$ & $\begin{array}{l}\text { Child age and sex, maternal and } \\
\text { parental education, maternal } \\
\text { occupation, socioeconomic } \\
\text { status, sibling at birth, smoking } \\
\text { and alcohol consumption } \\
\text { during pregnancy, duration of } \\
\text { breastfeeding, BC concentrations } \\
\text { at home, traffic noise annoyance } \\
\text { at home, home tobacco use, the } \\
\text { Urban Vulnerability Index at home } \\
\text { address, and the area level }\end{array}$ \\
\hline Grineski et al. (54) & $\begin{array}{l}\text { El Paso, Texas, } \\
\text { USA }\end{array}$ & Cross-sectional & $\begin{array}{l}\text { Caretakers of } \\
1888 \text { fourth } \\
\text { and fifth grade } \\
\text { children in the } \\
\text { EPISD }\end{array}$ & $\begin{array}{l}\text { School-based } \\
\text { exposure to } \\
\text { hazardous air } \\
\text { pollutants }\end{array}$ & $\begin{array}{l}\text { Impact of individual } \\
\text { children's academic } \\
\text { performance (Grade } \\
\text { point average) }\end{array}$ & $\begin{array}{l}\text { An interquartile range increase in school-level } \\
\text { HAP exposure was associated with an adjusted } \\
0.11-0.40 \text {-point decrease in individual students' grade } \\
\text { point averages (GPAs). Non-road mobile and total } \\
\text { respiratory risk had the largest effects on children's GPA } \\
\text { of all HAP variables studied }\end{array}$ & $\begin{array}{l}\text { Age, sex, race/ethnicity, English } \\
\text { proficiency, mother's education, } \\
\text { economic deprivation, percent } \\
\text { of students economically } \\
\text { disadvantaged and student- } \\
\text { teacher ratio }\end{array}$ \\
\hline Guxens et al. (87) & $\begin{array}{l}\text { Europe (Sweden, } \\
\text { Netherlands, Italy } \\
\text { and Spain) }\end{array}$ & $\begin{array}{l}\text { Population- } \\
\text { based cohort }\end{array}$ & 8079 children & $\begin{array}{l}\text { Exposure to air } \\
\text { pollution during } \\
\text { pregnancy }\end{array}$ & $\begin{array}{l}\text { Childhood autistic } \\
\text { traits in the general } \\
\text { population }\end{array}$ & $\begin{array}{l}\text { Prenatal air pollution exposure was not associated with } \\
\text { autistic traits within the borderline/clinical range (odds } \\
\text { ratio }=0.94 ; 95 \% \mathrm{Cl}: 0.81-1.10 \text { per each } 10-\mu \mathrm{g} / \mathrm{m} 3 \\
\text { increase in NO2 pregnancy levels). Similar results were } \\
\text { observed in the different cohorts, for the other pollutants, } \\
\text { and in assessments of children with autistic traits within } \\
\text { the clinical range or children with autistic traits as a } \\
\text { quantitative score }\end{array}$ & $\begin{array}{l}\text { Age at delivery, education level, } \\
\text { country of birth, prenatal smoking, } \\
\text { parity, maternal height, pre- } \\
\text { pregnancy weight, child sex and } \\
\text { date of birth, and child's age at } \\
\text { autistic trait assessment }\end{array}$ \\
\hline
\end{tabular}




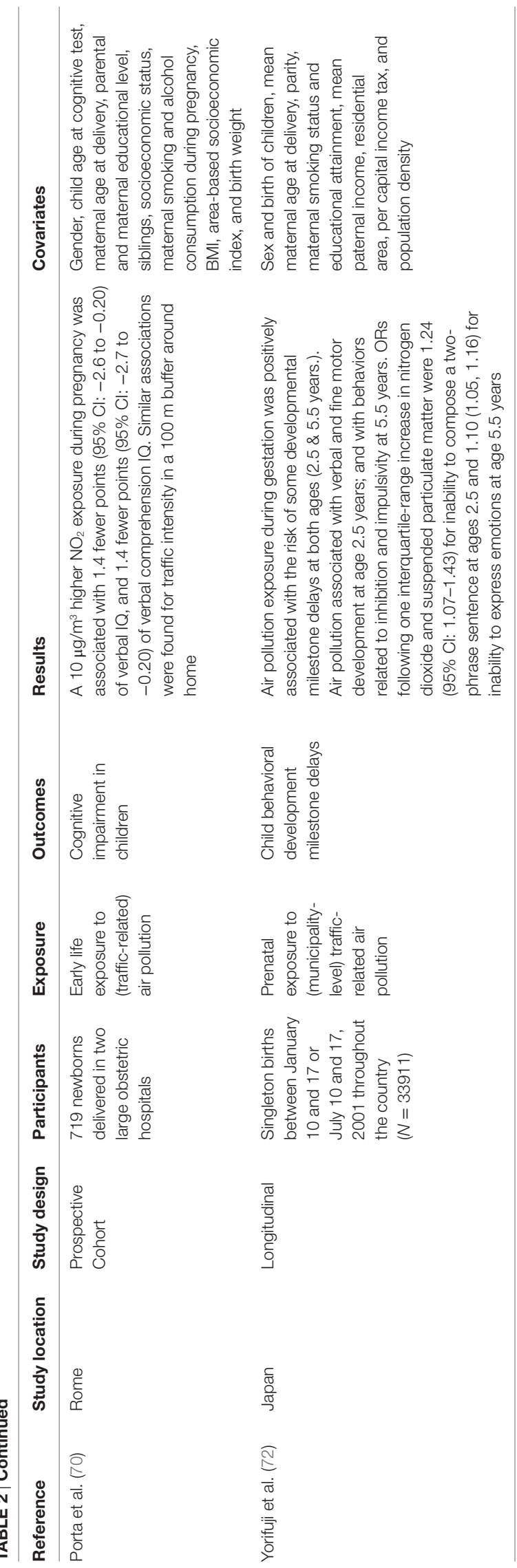

few existing studies suffer from small sample size issues because it is not feasible to conduct brain histological studies at a population level.

\section{Effects on Neuropsychological Function Evidence from the Cross-Sectional Studies}

Studies for neuropsychological effects have been more popular and vary in study design and methodology. Several cross-sectional studies have examined the associations between ambient or TRAP and neurobehavioral functions or neurological symptoms in adults. They suggest higher exposure to air pollution is associated with lower performance in neuropsychological tests. For example, Inserra et al. (33) examined the long-term health effects of ambient hydrogen sulfide $\left(\mathrm{H}_{2} \mathrm{~S}\right)$ exposure - determined by universal Kriging of known observations from air monitors - on neurobehavioral performance among 335 residents in more clearly defined exposed ( $>90 \mathrm{bbp}$ ) and unexposed ( $<50 \mathrm{bbp})$ neighborhoods in Dakota City, Nebraska. This study reported that after adjusting for age, gender, and testing language in a multivariate logistic regression model, poorer performance on a test of memory was marginally associated with high $\mathrm{H}_{2} \mathrm{~S}$-exposed residence [odds ratio $(\mathrm{OR})=0.6,95 \%$ confidence interval $(\mathrm{CI})=0.4-1.0]$, while other neurobehavioral functions (e.g., motor response, mood/affect) were not associated with exposure (33). In addition to having only marginally significant lower odds of having better memory test performance in the exposed group, the study might have suffered non-response bias. For example, not only the generalizability of the study was threatened by the fact that participation rate was only less than $75 \%$, non-respondent rate was higher in the unexposed group. This could have biased the results either toward or away from the null depending on the characteristics of non-respondents. Most importantly, this study is also subjected to exposure misclassification bias because not only it estimates exposure indirectly using statistical model but it also ignores personal activity patterns, which could have influenced personal exposures. As results, people who live in low exposed neighborhood may in fact have high exposure levels, and vice versa.

Chen and Schwartz (31) also examined neurobehavioral effects of long-term exposure to ambient PM and ozone in adults using the Third National Health and Nutrition Examination Survey (NHANES III) conducted in 1988-1994. Exposure was estimated using distance-weighted averages from all monitors in the residing and adjoining counties. After adjustment for important confounding variables, such as sociodemographic factors, lifestyle factors, medical risk factors, place of residence, and indoor pollution a in multiple linear regression analysis, each 10-ppm increase in annual ozone exposure was associated with increased symbol-digit substitution test score (SDST) and serial-digit learning test (SDLT) by 0.12 (95\% CI: $0.01-0.23)$ and 0.57 (95\% CI $0.08-1.06)$, respectively. Since highly exposed individuals had higher scores, indicating slower reaction time, these findings suggest adverse neurobehavioral effects of ambient air pollutants in adults (31). No significant findings were observed for $\mathrm{PM}_{10}$. In this study, the exposure assessment only uses one-time residential information based on environmental monitoring data; therefore, it might not have captured an accurate exposure measurement 
for individuals, leading to possibly inaccurate estimate of risk. In addition, the cross-sectional nature of the study prevents a causal interpretation due to the lack of temporality, although it is not likely that people with lower test scores chose to live in area with higher ozone concentration.

Ailshire and Crimmins (29) examined the cross-sectional association between residential concentrations of PM with aerodynamic diameter of $2.5 \mu \mathrm{m}$ or less $\left(\mathrm{PM}_{2.5}\right)$ and cognitive function in older adults using multilevel linear regression models. Older adults living in areas with higher $\mathrm{PM}_{2.5}$ concentrations had worse cognitive function ( $\beta=-0.26,95 \% \mathrm{CI}$ : -0.47 to -0.05$)$ even after adjustment for community- and individual-level social and economic characteristics. The strengths of the study are including a large, nationally representative sample of US older adults and considering individual and neighborhood confounders (29). Ailshire and Clarke (28) also conducted a similar cross-sectional association on neighborhood-level exposure to $\mathrm{PM}_{2.5}$ and cognitive function in a diverse, national sample of older U.S. adults $(n=780)$. The study found that those living in areas with greater exposure to $\mathrm{PM}_{2.5}$ had an error rate 1.5 times greater than those exposed to lower $\mathrm{PM}_{2.5}$ concentrations (IRR $=1.53,95 \% \mathrm{CI}$ : $1.02-2.30$ ) for $10-\mu \mathrm{g} / \mathrm{m}^{3}$ increments (28). For these two studies, the limitations include lacking of some components of cognitive assessments; neighborhood based measure of pollution may not fully apprehend individual-level exposure; being unable to determine long-term exposure to air pollution on cognitive function; missing other potentially important confounders, such as diet and lifestyle factors.

Gatto et al. (32) also examined cross-sectional associations between various ambient air pollutants $\left(\mathrm{O}_{3}, \mathrm{PM}_{2.5}\right.$, and $\left.\mathrm{NO}_{2}\right)$ and cognitive function among 1,496 adults (mean age 60.5 years) living in the Los Angeles Basin using regression model and found that $10-\mu \mathrm{g} / \mathrm{m}^{3}$ increases in $\mathrm{PM}_{2.5}$ was significantly associated with lower verbal learning ( $\beta=-0.32,95 \% \mathrm{CI}$ : -0.63 to $0.00 ; p=0.05)$ and significant association was observed with global cognition, after adjusting for age, gender, race, education, income, study, and mood. $\mathrm{NO}_{2}$ exposure $>20 \mathrm{ppb}$ and $\mathrm{O}_{3} \leq 34 \mathrm{ppb}$ was related with lower logical ( $\beta=-0.62,95 \% \mathrm{CI}:-1.35$ to 0.11 , compared to $\leq 10 \mathrm{ppb})$ and executive function $(\beta=-0.66,95 \% \mathrm{CI}:-1.35$ to $0.03)$. The strength of this study provides addition evidence of the effects of gaseous air pollutants on cognitive function. However, measurement error in exposure assignments and a feature of cross-sectional study design limit this study (32).

Recently, Tzivian et al. (38) conducted another cross-sectional study of adverse effects of long-term air pollution on cognitive functions [mild cognitive impairment (MCI), amnestic MCI (aMCI) and non-amnestic MCI (naMCI)] among adults aged 45-75 years in highly urban German Ruhr area. Cognitive functions were assessed at 5 year follow-up examination of the population-based Heinz Nixdorf Recall study and exposure assessment used the LUR model. The study found that long-term exposure to air pollution were associated with MCI, particularly aMCI $\left(\mathrm{PM}_{2.5}: \mathrm{OR}=1.22,95 \% \mathrm{CI}: 1.08-1.38\right)$. The main strength of this study is its capability to distinguish the effects of TRAP from traffic noise. In addition, large study sample, standard assessment method for outcome measurement, and availability of other confounders, such as lifestyle factors and medical history, are other strengths. However, exposure misclassification and cross-sectional study feature limit the value of this study in the causal inference (38).

\section{Evidence from the Cohort Studies}

To address the weakness of cross-sectional studies, several cohort studies have been conducted to examine the effects of air pollution exposure in the period preceding cognitive testing. For example, Ranft et al. examined the health effects of long-term exposure to TRAP on cognitive impairment in elderly women aged 68-79 years in Germany. The study found that living near busy streets within $50 \mathrm{~m}$ was associated with mild cognitive impairment measured by the neuropsychological test battery CERAD-Plus test, stroop test, and sniffing test after adjusting for important covariates, such as patient demographic factors, lifestyle, and medical history, in linear regression models (36). The main limitation that one needs to notice is the fact that although only people who lived in the same area for 20 years or more are included, activity patterns were not adjusted for. Residential exposure might not be accurate exposure measurement since people could spend most of their time elsewhere that has different exposure levels. A similar study in the U.S. also found similar results with significantly decreasing cognitive function scores with closer proximity to major road ways after adjustment for important covariates (Table 1) (39).

In addition, the effects of exposure to $\mathrm{PM}_{2.5-10}$ on cognitive decline were recently evaluated among 19,409 older women aged 70-81 years, who were part of the Nurses' Health Study cohort study (40). Recent and 7-14 years exposures to PM preceding baseline cognitive assessment were estimated using spatiotemporal smoothing models with the US EPA air quality monitoring system. This study found that higher long-term exposure to both $\mathrm{PM}_{2.5-10}$ and $\mathrm{PM}_{2.5}$ are associated with faster two-year cognitive declines after adjusting for important covariates in a generalized estimating equation regression model (Table 1). Despite significant evidence, similar to the previous study, the results of this study might be biased by exposure misclassification since estimation was indirect using spatial modeling, which may not adequately adjust for personal activity patterns.

Loop et al. examines the 1-year mean $\mathrm{PM}_{2.5}$ concentration preceding the cognitive assessment that was measured over the telephone using the Six-Item Screener (SIS) in a biracial, bigender national cohort $(n=20,150)$ of at least 45 years of age. The study found that only $8 \%$ were classified as the cognitively impaired in their most recent follow-up. A $10-\mu \mathrm{g} / \mathrm{m}^{3}$ increase in $\mathrm{PM}_{2.5}$ concentration was not reliably associated with an increased odd of incident impairment (1.26, 95\% CI: 0.97-1.64), but was slightly associated with incident impairment in urban areas (1.40, 95\% CI: 1.06-1.85). The strengths of the study include a nationwide and demographically diverse US cohort and satellite measurements for air pollution exposure assessment. Its major limitations could be a potential of systemic misclassification of outcome and exposure measurements and irrelevant windows of exposure (34).

Tonne et al. investigated the association between exposures to particulate air pollution (characterized by size and source) and a cognitive battery composed of tests of reasoning, memory, 
phonemic and semantic fluency, among the participants from the Whitehall II longitudinal cohort study $(n=2867)$. All particle metrics were associated with lower scores in reasoning and memory measured in the 2007-2009 wave but not with lower verbal score. Higher $\mathrm{PM}_{2.5}$ of $1.1 \mu \mathrm{g} / \mathrm{m}^{3}$ (lag 4) was associated with a 0.03 ( $95 \%$ CI: -0.06 to 0.002$) 5$-year decline in standardized memory score and a $0.04(-0.07$ to -0.01$)$ decline when restricted to participants remaining in London between study waves. This study provides support for an association between particulate air pollution and some measures of cognitive function, as well as decline over time in cognition; however, it does not support the hypothesis that traffic-related particles are more strongly associated with cognitive function than particles from all sources. This study included relatively large population cohort residing in largest city of Europe and explored various windows of exposure preceding cognitive testing (37).

\section{Evidence on Chemical Constituents of PM}

Studies of the effects of chemical constituents of PM on cognitive function in adults are limited. Only two studies have been found. One study reported that black carbon (BC) exposure was associated with declined cognitive functions among older men in another recent study (35). In their study, Power et al. found that for each doubling in daily average BC concentration measured by LUR models based on local monitor sites, the odds of having mini-mental status examination (MMSE) scores under 25, which indicates cognitive impairment, increases by $30 \%$ after adjustment for important covariates (Table 1). Each doubling in BC concentration was also associated with a 0.054 SD lower cognitive test scores ( $95 \% \mathrm{CI},-0.103$ to -0.006$)$. This study, however, also used indirect exposure estimation through mathematical models and did not adjust for personal activity patterns among adult elderly subjects.

The other cross-sectional study examined the effects of airborne manganese $(\mathrm{Mn})$ from industrial sources on cognitive function of adults residing in two Ohio towns. Significant relationships $(p<0.05)$ between Mn exposure and performance on working and visuospatial memory (e.g., Rey-O Immediate $b=0.19$, Rey-O Delayed $b=0.16$ ) and verbal skills (e.g., Similarities $b=0.19$ ) were found. The strength of the study includes use of stringent selection criteria as participants with prior or present occupational exposures to chemicals or neurotoxic agents at work were excluded; tests were administered by clinically trained administers and use of advanced modeling. The limitations of the study are absence of personal sampling of each participant's exposure to $\mathrm{Mn}$; the estimation of $\mathrm{Mn}$ inhalation exposure varies depending on the Mn release characteristics in both towns and uncertainties in air dispersion modeling; and bias may occur as participants were not randomly selected in those towns (30).

Overall, studies investigating the effects of various air pollution measures on neuropsychological functions are more common; however, their methodologies vary significantly, leading to difficulty in direct comparison between studies. First, it is important to recognize that individual exposure may be very different from air pollution levels recorded by air monitors. In fact, none of the studies we reviewed have adjusted for personal activities patterns. Secondly, studies have focused on different air pollutants with different methods of assessment, most of which are indirect estimates using models or nearby air monitors. Therefore, although there is evidence on general effects of air pollution, this evidence is not for yet sufficient any one particular pollutant. In addition, the studies also used various neuropsychological tests for the same construct, which make comparisons difficult. Moreover, lifetime air pollution exposure assessment preceding cognitive testing is challenge. Research on more appropriate methods is needed.

Despite weaknesses and some mixed findings, there is balance of evidence suggesting that air pollution has adverse effects of neuropsychological functions as measured by test scores in adults. More research is still warranted given the heterogeneity of current research.

\section{Effects on Neurodegenerative Disorders}

Several studies have been conducted to examine the effects of air pollution or TRAP on neurodegenerative disorders, such as Parkinson's disease, dementia, Alzheimer's disease, and others. Finkelstein and Jerrett, in a retrospective cohort study, investigated the association between Parkinson's disease and ambient air $\mathrm{Mn}$ in a cohort of 110,000 subjects in the cities of Toronto and Hamilton, Canada. Ambient Mn exposure was estimated by the Mn fraction of total suspended particulate (TSP) using two directly interpolated surfaces of TSP and Mn-enrichment factor. In Hamilton, the OR of Parkinson's disease was found to be 1.034 (95\% CI: $1.00-1.07$ ) per $10 \mathrm{ng} / \mathrm{m}^{3}$ increase in Mn in TSP after controlling for sex and birth year group in multiple logistic regression models (43). No significant differences were observed in Toronto. A closer analysis of age suggested that exposure to Mn advances the age of diagnosis of Parkinson's disease, indicating a significant negative effect. Although the magnitude of effect is relatively small, it might suggest clinical significance since Parkinson's is a serious disease that urgently needs intervention reduction of risk. In addition, the lack of residential mobility adjustment and the fact that interpolation models for exposure assessment might not have adequately represented personal exposure could have affected the results. Despite these potential limitations and mixed results for two cities, this study has shown some evidence toward the negative effects of pollution on Parkinson's disease.

Chang et al. evaluated the effects of air pollution on the risk of dementia using the data obtained from the National Health Insurance Research Database (NHIRD) of Taiwan, including those diagnosed with dementia between 2000 and 2010. Yearly average concentrations of pollutants were calculated from the baseline to the date of dementia occurrence, withdrawal of patients, or the end of the study. This study indicated that exposures to $\mathrm{NO}_{2}$ and $\mathrm{CO}$ were associated with an increased risk of dementia in the Taiwanese population. The limitations of this study include potential biases due to retrospective nature, unknown confounders, and unavailable relevant clinical variables since NHIRD data are anonymous. Large sample size, long follow-up period, and the reliability and accuracy of diagnosis and codes of dementia from the NHIRD are considered as the strengths of this study (42). Oudin et al. also conducted a prospective cohort study to examine the effects of air pollution on dementia in Northern Sweden. They also found that participants with highest TRAP exposure was associated with dementia diagnosis (hazard ratio 
(HR): 1.43, 95\% CI: 0.998-2.05) compared with those with lowest exposure. The study used the high quality data from the Betula study. However, this study did not consider the effects of traffic noise, which is highly correlated with TRAP (47).

Heydarpour et al. examined the spatial distribution of prevalent multiple sclerosis (MS) cases and their association with the spatial patterns of air pollution assessed with the previously developed LUR models. This study suggests that there was a statistically significant difference in exposure to $\mathrm{PM}_{10}, \mathrm{SO}_{2}, \mathrm{NO}_{2}$, and NOx $(p<0.001)$ in MS cases compared with controls. One of the main limitations of the study design is that no confounder was considered in the analysis (44). Malek et al. conducted a casecontrol study (51 cases, 51 controls) to examine the associations between suspected HAPs exposure and ALS in 6 counties near Philadelphia, from 2008 to 2011. Exposure to HAPs was assessed using residential census tract level data from the U.S. EPA National-Scale Air Toxics Assessment (NATA) data. This study showed that residential exposure to ambient air aromatic solvents was associated with increasing risk of ALS in 2002 (OR: 5.03, 95\% CI: 1.29-19.53) and 1999 (OR: 4.27, 95\% CI: 1.09-16.79). The study had a small sample size and exposure misclassification due to ecological measurements of exposure to HAPs and residential change (46). Angelici et al. examined the association of exposure to airborne PM on the occurrence of MS-related hospitalizations in Italy during 2002-2009. A total of 8287 MS-related hospitalization was obtained through discharge records and exposure to air pollutants was measured via air monitoring stations. Using Poisson regression, it was found that higher risk of MS hospital admission was associated with exposure to $\mathrm{PM}_{10}$ and the highest effect was observed from 0 to 7 days. A major limitation of the study is the feature of ecological study design (41).

Jung et al. conducted a cohort study of 95,690 individuals' age $\geq 65$ years old during 2001-2010 to determine the association between newly diagnosed Alzheimer disease and long-term exposure to ozone $\left(\mathrm{O}_{3}\right)$ as well as $\mathrm{PM}_{2.5}$ in Taiwan. The findings suggest that long-term exposure to $\mathrm{O}_{3}$ and $\mathrm{PM}_{2.5}$ above the current US EPA standards were associated with increased the risk of Alzheimer disease. The strengths of the study include the use of population-based database; of the use of newly diagnosed cases; and the first study conducted in Asian population. However, the major limitations include exposure errors of air pollution assessment and inadequate controlling of confounding (45).

Overall, the studies of air pollution and neurodegenerative disorders are limited. Existing studies have the limitations of poor air pollution exposure assessment and inadequate control of confounders. Relevant windows of exposure, more studies are needed to determine the association between air pollution and neurologically related disorders, such as Parkinson's, Huntington, and Alzheimer's diseases with appropriate methods.

\section{Effects on Other Non-Specific Neurological Symptoms}

A cross-sectional study examined the relationship between ambient ozone and building-related symptoms based on the U.S. EPA's Building Assessment Survey and Evaluation Study data. Ambient ozone level was estimated using the nearest US EPA local air monitors. This study found that neurological symptoms of fatigue and trouble concentrating were significantly associated with ambient ozone $\left(\mathrm{OR}=1.03,95 \% \mathrm{CI}: 1.01-1.05\right.$ per $10 \mu \mathrm{g} / \mathrm{m}^{3}$ increase in late workday ambient ozone) after adjusting for personal, workplace, and environmental variables, such as age, gender, smoking status, allergy, thermal exposure, humidity, and season in logistic regression models. Headache was also associated with ambient ozone $\left(\mathrm{OR}=1.03,95 \% \mathrm{CI}: 1.00-1.05\right.$ per $10 \mu \mathrm{g} / \mathrm{m}^{3}$ increase in late workday ambient ozone) after controlling for the same covariates previously mentioned (48). Although the ORs for association between ozone and neurological symptoms and headache are statistically significant, it is worth noticing that they are relatively small in magnitude. This study is limited by the cross-sectional design, which does not provide information on whether exposure precedes outcome or vice versa. In addition, the fact that ozone exposure was estimated using air monitors at varying distance away from buildings could limits the accuracy of exposure measurement. Furthermore, using study space averages of environmental exposure to assign to individual exposure could also hinder accuracy because it does not take into account personal activities.

Epidemiological evidence on adverse health effects of ambient air pollution on the nervous system in adults is generally limited. However, given what is available, evidence from the reviewed studies - regardless of study design - generally suggest that ambient air pollution has potentially adverse effects on neurobehavioral functions. However, it warrants further investigation with more advanced study designs, such as more cohort studies that allow for examination of temporal sequence, and better exposure assessment for more accurate estimation of risk. Since most studies reviewed above [except Ranft et al. (36), Chen and Schwartz (31), and Weuve et al. (40), which adjusted for education and income] were not able to adjust for community level factors (e.g., median income, housing status, etc.) and the gene-environment interaction - two factors which are known to be associated with both exposure and outcome - it is very important for future studies to also adjust for these factors to get more accurate estimation of effects $(92,93)$. Studies that show statistically significant effects of air pollution generally show weak magnitude of association. This could be because of the inability to control for these factors, which could be strong confounders since they may have stronger effect on neurobehavioral outcomes compared to air pollution. Furthermore, as previously mentioned, methodologies, including pollutant of interest, exposure assessment, and outcome assessment, vary between studies. This makes it difficult to directly compare results.

\section{Air Pollution and Neurotoxicity in Children Effects on Brain Biology and Histology}

Similar to adults, studies that examine the effects of air pollution on brain histology and biology in children are rare. This is perhaps due to the complex nature of study procedures and the difficulty in obtaining participants, especially for population level studies. Three studies were conducted by the same research group to evaluate the health effects of air pollution on brain abnormalities measured by magnetic resonance imaging (MRI), immunohistochemistry and electron microscopy among children in Mexico. Residency in a highly exposed area (Mexico city) was 
significantly associated with a higher proportion of prefrontal white matter hyperintense lesions compared to low exposed area (Polotitlán) in a hierarchical regression model adjusted for age and gender $(23,49)$. The pathological examination of olfactory bulbs (OB) pathology examined by immuno-histochemistry and electron microscopy also showed that the exposed groups exhibited immunoreactivity to $\mathrm{A} \beta 42$ and $\alpha$-synuclein and OB endothelial hyperplasia, and neuronal accumulation of particles, suggesting more neuroinflammation (22).

Despite positive findings, these three studies are limited from the cross-sectional design, small sample size, and the fact that important confounders (e.g., other health conditions for the first study, the latter study did not adjust for any confounder) were not adjusted for, making inference particularly weak. Most importantly, the independent variable in this study is not clearly indicated, suggesting that one cannot attribute the reported effects to pollution. Specifically, the authors have classified people in Mexico city as highly exposed while those in Polotitlán as control. This might have been subjected to misclassification of exposure because the level of exposure within city can vary depending on activity patterns and local variation.

Overall, evidence on the effects of pollution on brain biology and histology is still limited. Existing studies show evidence of adverse effects; however, are limited from methodological weaknesses, such as small sample size, lack of adjustment for potential confounders, poorly defined exposures, etc. More studies with more rigorous methods are still needed to yield clearer conclusion in this area.

\section{Effects on Child Neurodevelopment}

Studies for neuropsychological functions are more common. A total of 24 studies have been conducted to examine health effects of air pollution or TRAP during pregnancy and/or early childhood on neurodevelopment in children.

\section{Prenatal Exposure to Air Pollution Alone}

Fetuses are more susceptible to the harmful effects of a variety of environmental contaminants. Prenatal exposure to air pollutants may produce a variety of neurodevelopmental problems as a result of irreversible nervous system damage. Due to this concern, several epidemiological studies have been developed to examine prenatal exposure to air pollution on early child neurodevelopment.

Perera et al. conducted a prospective cohort study to examine the effects of prenatal exposure to air polycyclic aromatic hydrocarbons (PAHs) on neurobehavioral disorders among 183 subjects. Personal monitoring during the third trimester of pregnancy was used to collect the air samples, which were analyzed for prenatal exposure to airborne PAHs, and the Bayley Scales of Infant Development-Revised version was used to assess mental and psychomotor development in the first 3 years of life. The study found that children in the upper quartile of exposure to PAHs during the third trimester scored 5.69 points lower using the mental development index than those in the lowest quartile of exposure to PAHs. In addition, the risk of cognitive developmental delay for high PAHs exposure group was almost three times compared to children in the low exposure group (69). The most important limitation this study has, as recognized by the authors, is the fact that while postnatal exposures and exposure during other times of pregnancy can potentially affect the outcome, they were not available for adequate adjustment. Similarly, Tang et al. evaluated the associations between prenatal exposure to ambient PAHs, lead, and mercury from combustion of coal and fossil fuels, and cognitive functions measured by the Gesell Developmental Schedules at 2 years of age among children in Tongliang and Chongqing, China. PAH-DNA adducts and lead and mercury in blood cord were measured as individual exposure to these chemicals. After adjusting for important confounders, increased PAH-DNA adducts was associated with decreased motor area developmental quotients (DQ) and average DQ (Table 2). The OR of developmental delay in motor area was 1.91 (95\% CI: 1.22-2.97) per 0.1 unit increase in PAH-DNA adduct (71). Similar to the previous study, the authors recognized that the lack of postnatal exposure could be problematic because these exposures may have effect on subsequent development. While the use of biomarkers for measurement of exposure is less subject to misclassification, studies like these could still be limited by the fact that it assumes peripheral biomarkers are accurate representations of what is going in the CNS. Nevertheless, with more rigorous methodology including prospective cohort design, more accurate exposure assessment via biomonitoring, and adjustment of potential confounders, the association between ambient air pollution and adverse neurological effects remains consistent. Lovasi et al. also examined the effects of prenatal exposure to $\mathrm{PAH}$ and neighborhood social context on cognitive test scores at 5 years of age measured by the Wechsler Preschool and Primary Scale of Intelligence-Revised (WPPSI-R) in 1998-2006. Prenatal $\mathrm{PAH}$ exposure during the third trimester of pregnancy was measured via personal monitoring. The study found that the prenatal PAH exposure were significantly associated with 3.5-point lower WPPSI-R total and 3.9-point lower verbal scores. Some strengths of this study include prospective design, the presence of data from prenatal personal $\mathrm{PAH}$, and neighborhood definition using $1-\mathrm{km}$ network buffers, etc. However, high-risk women before the third trimester and children who took Spanish version of the test scores were excluded from the study, thus, may result in selection bias (68). Another recent longitudinal study was also conduct to investigate the association between prenatal $\mathrm{PAH}$ exposures and cognitive dysfunction in 170 children in Krakow, Poland. The exposure was also assessed using the cord blood PAH-DNA adducts. Consistent findings with Tang's study were observed. This study is limited due to relatively smaller number of subjects in the study and the main strength is individual-level prenatal exposures to PAH, based on cord blood PAH-DNA adducts (66).

Guxens et al. examined the associations between prenatal exposure to residential air pollution assessed by LUR and infant mental development among 1889 children. The study also showed negative effects of exposure to ambient air pollution on neuropsychological outcomes measured by Bayley Scales of Infant Development. Specifically, the study showed that prenatal exposures to $\mathrm{NO}_{2}$ and benzene were inversely and significantly associated with infant mental development in the group of low intake of vegetable and fruit during pregnancy (64). In this study, loss to follow-up was more likely to be participants from lower 
socioeconomic class, which could lead to bias; however, in analyses, the authors included large sets of variables related to participation and results remained consistent across strata. Therefore, in addition to showing that prenatal exposure to residential air pollution negatively affects mental development, this study also shows potential effect modification of the level of antioxidant intake. In other words, antioxidant intake can alleviate the negative effects of air pollution on mental development. Furthermore, Guxens et al. examined prenatal exposure to outdoor air pollution and its effect on cognitive and psychomotor development in childhood $(n=9482)$ within six prospective cohort studies. The concentrations of air pollution were also calculated using LUR models, air monitoring campaigns and back-extrapolation procedure. Similarly, a negative association was observed between prenatal air pollution, especially $\mathrm{NO}_{2}$ in psychomotor development in children aged 1-6 years but no association was observed in cognitive development. The strength of this study provides evidence on long-term effects of prenatal exposure on neurodevelopment among the pooled samples of several large cohorts. The main limitations include the heterogeneity of outcome measurements from different cohort and exposure measurement errors (65).

In South Korea, a prospective birth cohort study was conducted to study the effects of prenatal exposure to air pollution $\left(\mathrm{PM}_{10}\right.$ and $\left.\mathrm{NO}_{2}\right)$ on neurodevelopment in early childhood (first 24 months). The neurodevelopment was assessed using Korean Bayley Scale of Infant Development II (K-BSID-II) at ages of 6, 12 , and 24 months and air pollutants exposure was measured using inverse distance weighting (IDW) model. Prenatal exposure to $\mathrm{PM}_{10}$ and $\mathrm{NO}_{2}$ was significantly associated with Mental Developmental Index and Psychomotor Developmental Index at 6 months of age, but no significance at 12 and 24 months of age. This study provided a longitudinal assessment of neurodevelopment at multiple ages while a relatively small sample size and air pollution exposure errors could limit the value of this study (67).

Cowell et al. investigated the effects of prenatal traffic-related BC exposure on children's memory and learning. Validated spatiotemporal LUR models were used to calculate BC exposure. The Wide Range Assessment of Memory and Learning-Second Edition (WRAML2) was used to assess memory functions in children at 6 years of age. It was found that boys born to mother with the highest prenatal stress and higher BC showed lower memory score. The major strengths of the study are its prospective study design and inclusion of mother-child pairs from the understudied group with exposure to both air pollutants and stress. Similarly, this study has the limitations of exposure errors and residual confounding (63).

Chiu et al. examined sensitive windows of prenatal exposure to $\mathrm{PM}_{2.5}$ on neurodevelopment of children. Individual prenatal exposure to $\mathrm{PM}_{2.5}$ was measured using hybrid satellite-based spatio-temporal resolved prediction model and children aged $6.5 \pm 0.98$ years were tested for neurodevelopment measurement. This study found that increased $\mathrm{PM}_{2.5}$ exposure in specific prenatal windows was associated with poorer functions across memory and attention domains. The strengths of this study include the use of satellite data for air pollution exposure assessment and distributed lag models to identify sensitive windows for effects on neurodevelopment (62).
A recent Japanese study was also conducted to examine the association between prenatal exposure to outdoor air pollution and delay in behavioral development in nationally representative (singleton births) children. Positive associations between air pollution and neurodevelopmental outcomes were observed. Several limitations include the use of untested survey questions and methods of outcome measurements, and the possibility of misclassification errors (72).

Porta et al. conducted a prospective cohort study of TRAP and cognitive function among 719 newborns enrolled in GASPII project in Rome, where cognitive development of 474 children at age 7 were scaled using Wechsler intelligence Scale for ChildrenIII and LUR model was used to assess exposure to $\mathrm{NO}_{2}, \mathrm{PM}_{\text {coarse, }}$, $\mathrm{PM}_{2.5}$ and $\mathrm{PM}_{2.5}$ absorbance at birth. This study showed that prenatal exposure to $\mathrm{NO}_{2}$ per $10 \mu \mathrm{g} / \mathrm{m}^{3}$ increase was inversely associated with the verbal IQ $(-1.4,95 \% \mathrm{CI}:-2.6,-0.20)$ and verbal comprehension index $(-1.4,95 \% \mathrm{CI}:-2.7,-0.20)$. In addition, it was also found that traffic intensity of all roads in a 100 -mt buffer and total traffic intensity of major roads in a 100mt buffer were inversely associated with full-scale IQ, Verbal IQ and Verbal comprehension index. The study uses standardized measures to calculate the exposure for each pollutant. The study was limited due to selection bias at the enrollment period as the study participants were reprehensive of more educated group and loss of follow-up was caused by socioeconomic status (SES) status (70).

Overall, existing evidence suggests that prenatal exposure to air pollutants may have impacts on child neurodevelopment regardless different study designs, study populations, air pollution exposure assessments, and outcome measurements. Although most of studies rely on the data from air monitors or statistical methods for air pollution exposure assessments, a few other studies have been conducted to investigate the neurological effects of exposure to ambient air pollution measured by more accurate and objective exposure assessment, such as biomarkers in children. More research on identifying sensitive windows and susceptible groups, application of objective exposure measurements, such as personal monitor, and intervention is required.

\section{Prenatal and/or Childhood Air Pollution Exposure}

Several studies have been conducted to assess the impacts of air pollution assessed at ecological levels, such as school or city levels, on cognitive functions in children. Calderón-Garcidueñas et al. investigated the effects of living in highly exposed city on cognitive functions among children measured by the Wechsler Intelligence Scale for Children-Revised. Residency in a highly exposed area (Mexico City) was significantly associated with cognitive deficit $(23,50)$. Furthermore, the same authors, in a subsequent study with the same exposure definition, examined the health effects of air pollution on olfactory dysfunction measured by the University of Pennsylvania Smell Identification Test (UPSIT). Results suggested that the highly exposed group had a significantly lower UPSIT score than the controls (22). As previously mentioned, these two studies are limited by sample size, poorly defined independent variable, and the lack of control for potential confounders. With larger sample size, a study conducted in China examined the health effects of TRAP 
on neurobehavioral functions among 282 third-grade children. A school in an area with severe TRAP was compared to another school from an area of low traffic density based on ambient air quality monitoring data. The level of traffic air pollution for the schools was determined by annual concentrations of nitrogen dioxide and $\mathrm{PM}_{10}$ measured by air monitors and on-site samplers. In multiple ordinal logistic regression models, after adjusting for other covariates, such as demographics, birth weight, delivery method, breast feeding, vision, familiarity with computer games, and household pollution, TRAP exposure was significantly associated with poorer performance on neurobehavioral tests (Table 2) (61). Van Kempen et al. conducted a cross-sectional study to investigate the association between air pollution and transportation noise on the cognitive performance from 553 children from 24 primary schools and in home setting, using multilevel modeling and found that $\mathrm{NO}_{2}$ exposure at school was statistically significantly with memory span length during DMST $\left(x^{2}=6.8, \mathrm{df}=1, p=0.01\right)$. The combined interaction between air pollution and traffic noise at school was significantly associated with "block" condition of SAT; and at home, it was significantly associated with SRTT, Simple Reaction Time Test and 'arrow' condition of SAT (60). A prospective cohort study was conducted to examine the effects of TRAP exposure on cognitive development among 2715 children aged 7-10 years from 39 schools in Barcelona. TRAP at school was assessed. This study showed smaller growth in cognitive development in children at schools highly exposed to TRAP (7.4\%, 95\% CI: 5.6-8.8\%) than low exposed schools (11.5\%, 95\% CI: 8.9-12.5\%). Similarly, children at schools highly exposed to elemental carbon (EC), $\mathrm{NO}_{2}$, ultrafine particle number (UFP) had smaller improvements in all the cognitive parameters. (59). Grineski et al. also conducted a cross-sectional study to examine the impacts of school-level exposures to HAPs on academic performance at fourth and fifth grade children in the El Paso (Texas, USA). In multilevel models, HAP exposures at school were associated with lower individuallevel grade point (54). Aside from previously mentioned weaknesses, such as inability to adjust for personal exposure and activity patterns, these studies are limited due to an ecologic exposure assessment.

In addition, a few cross-sectional studies have been conducted to provide useful information in this area. Kicinski et al. examined the association between traffic exposure and neurobehavioral performance in 606 adolescents in Belgium. Urinary levels of trans, trans-muconic acid, and the distance-weighted traffic density were used to estimated traffic exposures. The study indicated exposure to air pollutants was inversely associated with sustained attention (95\% CI: -0.51 to -0.02 ). Similarly, no significant association was observed in traffic exposure and neurobehavioral domains. The study had some limitations, including a cross-sectional feature, the use of irrelevant urine samples as they weren't collected at the time of examination, and the possibility of misclassification bias (56). In Spain, Forns et al. also conducted a cross-sectional study to examine the associations of exposure to TRAP (EC, BC and $\mathrm{NO}_{2}$ ) and noise at school on behavioral development of schoolchildren aged 7-11 years during 2012-2013. Forns et al. found similar results as described by Van Kempen et al. However, selection bias is one of concerns regarding this study (52). Overall, these studies limit their ability for causal inference due to the feature of cross-sectional study design.

Cohort study design has also been applied to study the impacts of air pollution on cognitive function in children. Suglia et al. examined the relationship between BC, measured by LUR models, and cognitive functions among 202 children in Boston, Massachusetts. Cognitive functions were measured by the Kaufman Brief Intelligence Test (KBIT) and the Wide Range Assessment of Memory and Learning (WRAML). After adjustment for age, gender, primary language spoken at home, mother's education, birth weight, passive smoking, and blood lead levels, BC exposure was associated with a decreased scores in various cognitive measures (see Table 2) (58). Despite consistent association between exposure to pollution and risk of lower cognitive scores, results should be carefully interpreted. Specifically, this study assessed exposure at home residence while ignoring exposure at school/work at which participants might spend a considerable time, leading to inaccurate estimation of personal exposure and limited inference. Friere et al., in a cohort study investigated the effects of $\mathrm{NO}_{2}$ exposure on various neuropsychological constructs, such as general cognition, quantitative and working memory, and gross motor. Exposure assessment is more specific in this study and was measured by LUR model. Children exposed to higher $\mathrm{NO}_{2}\left(>24.75 \mathrm{mg} / \mathrm{m}^{3}\right)$, compared to their counterparts, have a statistically significant decrease of 8.61 points in gross motor score, while there is no significant statistical differences for the other three constructs, which could likely be due to low sample size with too many covariates in analyses (Table 2) (53). Lin et al. recruited 533 mother-infant pairs from within 11 towns in Taiwan to study the effects of ambient air pollution on neurobehavioral development during pre and postnatal stages. This study found that increased $\mathrm{SO}_{2}$ exposure during pregnancy and post pregnancy (up to 1 year) was significantly associated with decrease in fine motor performance at 18 months Similar to other studies, residual confounding and exposure error are the main weaknesses of this study (57). Harris et al. studied associations between gestational and childhood exposures to TRAP and childhood cognition among a prospective cohort of 1,109 mother-child pairs in USA. The exposure to $\mathrm{BC}$ and $\mathrm{PM}_{2.5}$ were estimated using both residential proximity and validated spatiotemporal LUR models. The cognitive development was estimated using KBIT-2 at mean age of 8 years. Using linear regression model, lower non-verbal IQ (-7.5 points, 95\% CI: -13.1 to -1.9 ), verbal IQ ( -3.8 points, $95 \% \mathrm{CI}:-8.2$ to 0.6$)$ and visual motor ( -5.3 points; $95 \%$ CI: -11.0 to 0.4 ) scores were associated with residential proximity - $50 \mathrm{~m}$ away major roadway at birth than those living in $\geq 200 \mathrm{~m}$ away from roadway. $\mathrm{PM}_{2.5}$ exposure at third trimester had no association with poor cognition. One of the major limitations of this study is measure errors of exposure assessment (55). Cohort study design has the advantage of causal inference. However, similar to many studies discussed before, these studies are potentially subjected to exposure misclassification bias. More specifically, while children are not stationary, e.g., exposures were estimated using levels predicted at their home; therefore, this estimation may not reflect true exposure. 


\section{Effects on Neurodevelopmental Disorders}

The potential impacts of air pollution on neurodevelopmental disorders, such as autism and attention deficit and hyperactivity disorder (ADHD), have arisen a great concern. Evidence on the association between air pollution and neurodevelopmental disorders has dramatically increased in recent years. To better test the causal relationship, several cohort and case-control studies have been conducted to examine the relationship between ambient air pollution and neurodevelopmental disorders in children.

\section{Autism Spectrum Disorder}

Windham et al. investigated the effects of HAPs on autistic spectrum disorder among children born in 1994 in San Francisco Bay area (76). Exposures to HAPs were determined by US EPA Gaussian air dispersion model. The study found that top quartiles of exposure to chlorinated solvents and heavy metals are associated with higher odds of having autistic spectrum disorder among children after adjusting for important covariates (Table 2). This study, however, is limited by the fact that exposure misclassification is possible due to indirect estimation by air dispersion model. More specifically, estimation of exposure at residential address using air monitoring sites cannot adequately control for personal activity patterns, leading to misclassification of exposure.

Kalkbrenner et al. also conducted a case-control study investigating the effect prenatal exposure to HAPs and autism spectrum disorder (ASD) among children at age eight (77). Exposure to ambient metals, PM, and volatile organic pollutants were estimated at the census-tract level of birth residence using the 1996 National Air Toxics Assessment annual-average model. This study found that prenatal exposure to air pollutants including quinoline $(\mathrm{OR}=1.4,95 \% \mathrm{CI}=1.0-2.2)$, and styrene $(\mathrm{OR}=1.8,95 \% \mathrm{CI}=1.0-3.1)$ were associated with elevated risk for autism at age eight after adjusting for important covariates (Table 2). Besides the marginally significant results, this study is also limited by several weaknesses. First, exposure misclassification is possible due to the lack of information on activity patterns. Second, the use of children with other developmental disorders as controls could have biased results towards the null because it is possible that children with developmental disorders in general are more exposed to pollution.

Volk et al., in their recent study investigating the effects of proximity to freeway on risks of autism, found that after controlling for demographics and maternal smoking status, multiple logistic regression models show that pregnant mothers living more than $309 \mathrm{~m}$ away from freeway have an $86 \%$ increased risk of having the child with autism ( $\mathrm{OR}=1.8695 \% \mathrm{CI}$ : 1.04-3.45). More importantly, this study also made an attempt to investigate time of exposure - which many studies for children we reviewed did not address - and found that exposure during third trimester is associated with 2.22 times the risk $(\mathrm{OR}=2.22$, 95\% CI 1.16-4.42) (78). Since this study requires accurate information on residential information during different trimester of pregnancy, recall bias could be a potential limitation.

Becerra et al. conducted a case-control study investigating the effects of TRAP exposure during pregnancy on development of autistic disorder (AD) among children born in 1995-2006 in
California. The prenatal exposures to air pollutants were measured using both the nearest air monitoring stations and a LUR model. The study indicated that $12-15 \%$ increases in odds of AD were associated with per inter quartile range increase for ozone (OR: 1.12, 95\% CI: 1.06-1.19; per 11.54-ppb increase) and PM $\leq 2.5 \mu \mathrm{m}$ (OR: 1.15 ; $95 \%$ CI: $1.06-1.24$; per $4.68-\mu \mathrm{g} / \mathrm{m}^{3}$ increase) after adjusting for both maternal and perinatal characteristics (includes SES). Selection bias may likely exist in this study (79).

Another prospective cohort study was used to investigate the associations between long-term exposure to air pollution and newly diagnostic ASD among 49,073 children $<3$ years in Taiwan. The results indicated that risk of newly diagnostic ASD was increased with an increase in ozone exposure (Adjusted HR: 1.59, 95\% CI: $1.42-1.78$ per 10 ppb increase); $\mathrm{CO}(\mathrm{HR}=1.37$, 95\% CI 1.31-1.44); NO2 ( $\mathrm{HR}=4.43,95 \%$ CI 3.33-5.90), and $\mathrm{SO} 2$ (HR $=1.18$, 95\% CI 1.09-1.28 per $1-$ ppb increase). Cohort study design, larger sample size and longer follow-up period are some strengths of the study. The main study limitation is potential residual confounding (80).

Roberts et al. also conducted a case-control study investigating the effect prenatal exposure to HAPs and ASD among children of participants in the Nurses' Health Study II (325 cases, 22,101 controls) and found that the perinatal exposures to highest quintile of diesel (highest OR: 2.0, 95\% CI: 1.0-4.0), lead (1.6, 95\% CI: 1.1-2.3), Mn (1.5, 95\% CI: 1.1-2.2), cadmium (1.5, 95\% CI: 1.0-2.1), and overall metals (1.6, 95\% CI: 1.1-2.4) were significantly associated with development of ASD compared the lowest quintile. Gender might modify the effects of HAPs on ASD. Despite large national sample size, this study is limited, such as inaccurate measurement of exposures and collection of unreliable prenatal residency information during or post pregnancy (81). Raz et al. also conducted a nested case-control study (245 cases and 1522 controls) to examine the association between maternal exposure to PM air pollution and ASD in the offspring of those participants in the Nurses' Health Study II. Diagnoses of ASD were based on maternal report and exposure to $\mathrm{PM}_{10}$ and $\mathrm{PM}_{2.5-10}$ was estimated with the validated spatiotemporal model. It was found that maternal exposure to $\mathrm{PM}_{2.5}$ during pregnancy was associated with greater odds of ASD in children. In addition to exposure misclassification due to lack of the exact date of residential change and activity pattern information, the participants as all nurses may limit the generalizability of this study (84).

Another case-control study (78) examined the effects of TRAP on autism (279 cases, 245 controls) among those enrolled in the Childhood Autism Risks from Genetics and the Environment (CHARGE) Study in California. The residential information was collected using questionnaire. TRAP exposure at residency was estimated using a line-source air quality dispersion model. This study found significant associations among cases living in highest quartile between TRAP exposure in first year of life $(\mathrm{OR}=3.10,95 \% \mathrm{CI}: 1.76-5.57)$, all pregnancy $(\mathrm{OR}=1.98,95 \%$ CI: $1.20-3.31)$, first trimester ( $\mathrm{OR}=1.85,95 \% \mathrm{CI}: 1.11-3.08)$, second trimester $(\mathrm{OR}=1.85,95 \% \mathrm{CI}: 1.11-3.08)$ and third trimester $(\mathrm{OR}=2.10,95 \% \mathrm{CI}: 1.27-3.51)$ and autism. This study is limited due to effect of confounding from relating factors, such as lifestyles, nutrition, other residential exposures, and proximity of physician diagnosis (82). 
Similarly, Kalkbrenner et al. studied the effects of exposure to $\mathrm{PM}_{10}$ on autism spectrum disorder (autism). A total of 645 cases in North Carolina and 334 cases in the San Francisco Bay area were selected. Children in the control group were randomly selected from birth certificate based on counties and birth years of the selected cases. Geo-statistical interpolation method was used to assess individual exposure to $\mathrm{PM}_{10}$ during within 3-month periods of preconception through the first year of life. This study indicates exposure $\mathrm{PM}_{10}$ during the third trimester of pregnancy was associated with autism. The study has enough sample size and was able to control for maternal socioeconomic factors, while it is lack of the data for other pollutants, such as $\mathrm{PM}_{2.5}$ and $\mathrm{NO}_{2}$, which have been studied in other studies (83).

A population-based case-control (217 cases, 226 controls) study was conducted to investigate the risk of ASD due to prenatal and early childhood exposure to $\mathrm{PM}_{2.5}$ among children born between 2005 and 2009 in six counties in Southwestern Pennsylvania $(85,86)$. Exposure to $\mathrm{PM}_{2.5}$ was estimated using the LUR. This study found that prenatal exposure and postnatal exposure to $\mathrm{PM}_{2.5}$ are significantly associated with increased risk of ASD. The strengths of this study include having residential histories for $\mathrm{PM}_{2.5}$ exposure assessment and several windows of exposure from 3 months prior to conception through the second year of life to be considered (85). Additionally, based on the same study subjects, the investigators also examined the association between ASD and HAPs. The levels of 30 selected neurotoxicants at census tracts of residential address were assessed using the 2005 US EPA NATA data. This study reported that mothers living in areas with higher levels of styrene and chromium during pregnancy had a higher risk of ASD among their offspring. The limitations of this study include a semi-ecological design that assumed that all individuals at the same census tract had the same exposure levels of HAPs and lack of longitudinal data of HAPs (86). Guxens et al. used four European population-based birth/child cohorts to examine the effects of prenatal air pollution exposure on childhood autistic traits among general population (>8000 children). LUR models were applied to estimate nitrogen oxides and PM at birth residency. This collaborative study found that prenatal exposure to $\mathrm{NO}_{2}$ and $\mathrm{PM}$ was not associated with autistic traits at ages of 4-10 years old in children. This study consists of large sample size subjects with prospective and longitudinal study design, considering it as the most important strength (87).

\section{Attention-Deficit Hyperactivity Disorder}

There were also a few studies which have been conducted to examine the effects of air pollution on ADHD. Siddique et al. in Delhi, India investigated the relationship between vehicular air pollution and ADHD in children through a cross-sectional study and has found a dose-response relationship between $\mathrm{PM}_{10}$ exposure and risk of ADHD in children after controlling for age, SES, and BMI in a logistic regression model. Exposure to outdoor air pollution was determined by fixed site monitoring stations, and was adjusted for indoor levels measured by battery operated monitors. This study is limited by two major factors: (a) the cross-sectional design prevents causal inference, and (b) exposure based on fixed monitor stations are likely subjected to misclassification as they are not reflective of personal exposure. Despite weaknesses, these findings show some evidence that ambient air pollution may have adverse neurologic effects relevant to many neurological disorders, such as ADHD (73).

Newman et al. investigated the association between elemental carbon attributed to traffic (ECAT) and ADHD symptoms at 7 years of age in a birth cohort from the Cincinnati Childhood Allergy and Air Pollution Study (CCAAPS). ECTA exposure during the first year of life was estimated based on the data from air monitoring stations and LUR modeling. Significant association was observed between exposure to high ECAT and at-risk score for hyperactivity (1.7, 95\% CI: 1.0-2.7) after controlling for sex, cigarette exposure during the first year of life and maternal education. In addition to it, significant increase in at risk scores for hyperactivity was associated with exposures to higher air pollutants among children born to mothers with higher education (1.7, 95\% CI: 1.0-2.7) but the sample size was limited to children born to mothers with higher education attainment. The positive association could be explained as, higher maternal education could increase the school achievement expectations in mothers, whose reports might be higher for their children behavioral concerns. The selection of study participants was based on high risk for atopy, which may limit the actual results (74).

Gong et al. conducted a cohort study on the effects of pre and postnatal exposures to TRAP and its association with ASD and ADHD among 3426 twin children born in Stockholm in 1992-2000. The neurodevelopment outcomes of the children were screened via telephone interviews and air pollution exposure assessment were assessed using residence time-weighted concentrations of $\mathrm{PM}_{10}$ and $\mathrm{NOx}$ based on $\mathrm{b}$ dispersion models. However, this study did not support an association between prenatal or postnatal air pollution and ASD or ADHD. Several factors could have grounds discrepancy in the study results, such as presence of considerably low level of pollution and effects of other unselected confounders, such as maternal smoking status, including SES variables might have led to inconsistent associations. The major study limitation includes probable inconsistency in neurodevelopment outcomes data obtained from those participating in CATSS and general population (75).

Overall, studies on the effects of pollution in young children have - similar to those for adults - lacked the adjustment of important confounders, such as community factors, genetics underpinnings, and more importantly, the investigation of a critical window of exposure. While it is difficult to prove the importance of a prenatal critical window of exposure due to the fact that diagnoses are often made long after birth, it is still informative to study the time during which exposure has the strongest association with neurologic health outcomes. Although several studies we reviewed have made some attempt to reveal critical period of exposure by measuring exposure at specific time during pregnancy (e.g., third trimester), it remains inconclusive. Furthermore, studies that examined neurocognitive functions used various tests, making comparison between studies difficult. There is a clear need to unify these methods in air pollution studies.

Although the number of epidemiological studies is still small and varies in methodology, evidence from cross-sectional 
studies, case-control studies, and cohort studies suggests that exposure to ambient or traffic air pollution could potentially cause adverse health effects on neurobehavioral functions in children. Especially, although the relationships between ambient air pollution and neurodevelopmental disorders, such as ADHD and autism, have been increasingly studied, evidence is still limited and inconsistent. Future research on this field may be needed as the increasing trends of these diseases over the past years.

\section{DISCUSSION}

As mentioned earlier, the fact that neurological effects ranked second out of the seventeen health effects of ambient air pollution suggests that ambient air pollution plays a critical role in neurologic health. Thus, the study of neurological effects of ambient air pollution is an important emerging field of environmental epidemiology. Our systematic review suggests that there is evidence linking ambient air pollution with adverse neurological effects in both adults and children. Overall, consistency was demonstrated across studies.

Given the relative infancy of this research area, most of the publications occurred over the last 5 years. A critical evaluation of the evidence is therefore timely, appropriate and necessary. The reported associations between air pollution and neurological effects will be discussed regarding the following aspects: measurement of exposures, confounding and outcomes, and biases. This evaluation is intended to help guide developing future studies in this field.

\section{Measurement of Exposure}

As direct associations between air pollution and adverse neurological effects need to be further established, exposure assessment of air pollution is a crucial element for future study. The effect of ambient air pollution on the nervous system is most likely to be long term or chronic in nature. With current technology typically used in population-based studies, measurement of exposure to air pollution is most likely to represent an exposure during a specific window in a lifetime. Challenges in the measurement of air pollution exposure include duration of exposure, residential and occupational stability, personal time-activity patterns, and temporal and spatial variation of air pollution. Many studies reviewed above used a binary exposure measurement, i.e., high polluted area vs. low polluted area, annual concentrations of air pollutants from surrounding monitors or distance to traffic ways as an estimate of air pollution. These exposure measurements can be problematic and misclassification of exposure is very likely. For this reason, it is crucial to develop more accurate methods to measure chronic exposure to air pollution in this field. In children, a few studies that we reviewed tried to remedy the issue by using a biomarker, such as PAH-DNA adduct level in cord blood (69) and personal monitoring (71).

\section{Biomonitoring}

Biomarkers, mainly DNA adducts measured by ${ }^{32} \mathrm{P}$-postlabelling (94) and PAH-DNA adducts level tested by enzyme-linked immunosorbent assays (95), are valuable to measure chronic exposure of air pollution. Studies suggest that DNA adducts level is highly associated with ambient air pollution (96-98). Only two of the many studies we reviewed used this method for exposure estimation $(66,71)$. However, even with these biomarkers, research still faces the difficulty of distinguishing the sources of exposure, which may also include exposures, such as cigarette smoking. For example, if a subject has high level of PAH-DNA adduct, it is possible to tell whether the person has high chronic exposure to the chemicals; however, it is not possible to discern whether the person is exposed through ingestion, inhalation or other routes of exposure. In addition, measuring DNA adducts level requires a blood sample, which are more invasive, timeconsuming and expensive than many population-based studies can accommodate. Another limitation regarding this method of exposure measurement is the fact that peripheral markers may not reflect the environment in the CNS. More importantly, since events in the CNS vary considerably by brain region, peripheral markers may not be very relevant in some cases.

\section{Personal Monitoring}

Personal monitors are often considered as the most accurate estimate of individual air pollution exposure. Among the studies we reviewed, only two used this method $(64,69)$. For most studies this type of measurement is not feasible due to high costs, a requirement of prospective cohort study design, and necessary length of measurement time. Nevertheless, studies in children may be promising given the flexibility in length of study time in children, particularly if examining a specific time window, such as prenatal period, which is a finite time period. Personal monitoring may provide an estimate of exposure less prone to misclassification than other methods of measuring air pollution exposure in children when a sufficient number of measures are taken in a specific window $(69,99,100)$. However, this method is very difficult to use in the adult population to measure life cumulative or chronic exposure to air pollution because the application of the method requires prospective study design, long length of follow-up, and a large sample size. Regardless of feasibility, given the lack of availability of reliable tools for measurement of exposure to air pollution, personal monitoring currently yields the best estimation of exposure and therefore should be considered as the current gold standard.

\section{Exposure Assessment by Modeling}

In this context, geographic information system techniques, such as LUR and other geo-statistical models, may complement personal monitoring and biomonitoring methods. In LUR model, various GIS parameters, such as traffic density, population density, elevation, and land use, are used to predict the small-scale spatial variation of pollutants $(101,102)$. Therefore, the model can be used to estimate concentrations at home locations based on the spatially refined independent variable datasets (103). This model is, however, known to capture spatial variability instead of temporal variability. The LUR model has mainly been used to examine long-term (e.g., over years or lifetime) exposure to air pollution $(104,105)$. Some studies we reviewed used the method of LUR models in the exposure assessment $(35,36,53,58)$. One of the limitations that these studies faced was estimating air pollution levels on current residential address, which ignores historic 
exposures. People are mobile and are not confined in the place where exposure was assessed leading to potential misclassification of exposure. In order to reduce the potential for exposure misclassification, efforts should be made to include information on individual residential history and duration of stay in LUR models. Subjects' activity pattern is also important and should be controlled for in statistical analysis.

\section{Critical Window of Exposure}

The timing of exposure to a neurotoxic agent is one of the most important factors determining the effects produced by a neurotoxic agent, particularly given the potential for accumulation over the life course. However, it is also possible that air pollution may affect the nervous system in other ways that can influence development of disease. Depending on the health outcome under study, there may be a limited window in which air pollution can have adverse effects or has stronger effects on the nervous system than other times in life, i.e., a critical or sensitive period (106). For example, prenatal exposures to different pollutants have been consistently found to have profound effects on postnatal life in infants $(64,69,71)$. Consequently, identifying a critical window of exposure during prenatal period is essential in determining whether there is a causal relationship between air pollution and the nervous system. Furthermore, the time window is important with respect to developing appropriate preventive strategies for reducing adverse neurological health effects of air pollution. However, research on this specific area regarding the neurological effects of air pollution is still limited, as suggested by the fact very few of the studies reviewed addressed this issue. In children, neurotoxic effects of prenatal and postnatal air pollution exposure are far less clear than other points in their life. Similarly, none of the research we reviewed has examined the susceptibility window of neurotoxicity of air pollution in adult populations.

While a critical window of exposure is important in understanding the effects of air pollution and to develop effective interventions, it is still important to recognize that it is difficult to attribute negative effects to only the critical window period. The problem lies in the fact that most health endpoints are diagnosed after birth, making it very difficult to rule early life effect and conclude that prenatal exposure is more relevant. Moreover, there is evidence that suggests acute effects of air pollution on different health outcomes, suggesting that acute effects might also be important for neurological endpoints (107-109).

\section{Confounding}

The nervous system is sensitive to multiple environmental exposures. A number of environmental neurotoxicants, such as lead, mercury, polychlorinated biphenyls, pesticides, and ionizing radiation, have been well reported (110-113). Therefore, controlling for the confounding effects of these factors is necessary for an accurate estimation of the health effects of air pollution on the nervous system. However, for some environmental pollutants, such as lead, air pollution is also one of the primary sources of exposure (114). A complete adjustment of the effects of chemicals may result in underestimating the health effects of air pollution because they may be the important components of air pollution. Under this circumstance, it becomes more complex to control for the confounding effects of these chemicals. Furthermore, as metal pollutants are highly correlated in most cases, the adjustment of all others in order to find the effect of one pollutant makes the multipollutant model extremely complicated. It may lead to higher chances of finding false negative results, due to over-adjustment. Moreover, since individual pollutants have been found to be affecting neurological outcomes as discussed throughout the paper, multiplicative and additive effects are possible; none of the study we reviewed has examined these interactions.

Confounding due to lifestyle factors, such as smoking and alcohol drinking, may also be problematic, as these factors are also associated with health effects on the nervous system and may also be associated with air pollution exposure, given correlations with living in disadvantaged neighborhoods $(115,116)$. Several studies have consistently reported that smoking including active smoking and passive smoking can cause cognitive impairment in both adults and children (117-120). However, other studies found that alcohol consumption was beneficial to cognitive functions in adults (121-124). Thus, the potential confounding effects of these factors are required to be considered differently when examining the health effects on the nervous system.

Recognizing environmental justice issues in this literature also warrant attention in terms of the potential confounding effects of individual-level and area-level socioeconomic position on the relationship between environmental exposures and health outcomes. For example, Miranda et al. have found that poorer and minority neighborhoods are more likely to experience higher pollution levels. In addition, socioeconomic factors are also highly correlated with higher disease burden. Despite the important need to adjust for these confounders, very few of the studies we reviewed adjusted for or examined effect modification by socioeconomic position of individuals or their communities (92).

Given that many health endpoints have genetic underpinnings, there is emerging yet another important confounding factor: gene-environment interaction (93). This suggests that there could be susceptible subpopulations that need more attention than others. However, none of the studies we reviewed addressed this issue, indicating that future studies in this field need to pay closer attention to gene-environmental interaction in order to make better inference, and to identify susceptible populations for prompt intervention.

\section{Publication Biases}

The studies reviewed above were all selected from publications in major journals being identified through electronic databases and manual searches. There is a possibility that studies with negative or null results are less likely to be published in major journals, especially in the relatively early stage of development of this topic $(125,126)$. Consequently, the results might point more toward a positive association. In addition, publications in non-English journals were not included in the review. Therefore, we cannot exclude the possibility that these studies could also impact the results of the review. However, given the recent interest in this topic, most studies are more likely to be at least presented at conferences and found in conference proceedings, of which we also searched. 


\section{CONCLUSION}

Despite being a new area of research, there is evidence of association between air pollution and neurological function in both adults and children. Air pollution continues to be important environmental and public health concerns worldwide. Since the literature on the association between the nervous system and ambient air pollution is relatively new, there is no doubt that more research is required to fully understand the relationships. Future studies might be designed to use more accurate exposure measurement, to target different health endpoints (e.g., endpoints measured by neuropsychological tests, neurodevelopmental disorders and neurodegenerative diseases), to pinpoint one specific toxicant of importance including gaseous air pollutants, and to emphasize the combined neurological effects of multiple air pollutants. Furthermore, identification of susceptible subpopulations is the priority in air pollution studies. Future studies should also be designed to investigate the potential effect modifiers including genetic factors of the neurotoxicity of air pollution. Identification of factors that

\section{REFERENCES}

1. Bernstein JA, Alexis N, Barnes C, Bernstein IL, Bernstein JA, Nel A, et al. Health effects of air pollution. J Allergy Clin Immunol (2004) 114:1116-23. doi:10.1016/j.jaci.2004.08.030

2. Dockery DW. Epidemiologic evidence of cardiovascular effects of particulate air pollution. Environ Health Perspect (2001) 109(Suppl 4):483-6. doi:10.1289/ehp.01109s4483

3. Patel MM, Miller RL. Air pollution and childhood asthma: recent advances and future directions. Curr Opin Pediatr (2009) 21:235-42. doi:10.1097/ MOP.0b013e3283267726

4. Dockery DW, Pope CA III. Acute respiratory effects of particulate air pollution. Annu Rev Public Health (1994) 15:107-32. doi:10.1146/annurev. pu.15.050194.000543

5. Xu X, Sharma RK, Talbott EO, Zborowski JV, Rager J, Arena VC, et al. PM10 air pollution exposure during pregnancy and term low birth weight in Allegheny County, PA, 1994-2000. Int Arch Occup Environ Health (2010) 84(3):251-7. doi:10.1007/s00420-010-0545-Z

6. Wang X, Ding H, Ryan L, Xu X. Association between air pollution and low birth weight: a community-based study. Environ Health Perspect (1997) 105:514-20. doi:10.1289/ehp.97105514

7. Darrow LA, Klein M, Flanders WD, Waller LA, Correa A, Marcus M, et al. Ambient air pollution and preterm birth: a time-series analysis. Epidemiology (2009) 20:689-98. doi:10.1097/EDE.0b013e3181a7128f

8. Parker JD, Mendola P, Woodruff TJ. Preterm birth after the Utah Valley Steel Mill closure: a natural experiment. Epidemiology (2008) 19:820-3. doi:10.1097/EDE.0b013e3181883d5d

9. Rich DQ, Demissie K, Lu SE, Kamat L, Wartenberg D, Rhoads GG. Ambient air pollutant concentrations during pregnancy and the risk of fetal growth restriction. J Epidemiol Community Health (2009) 63:488-96. doi:10.1136/ jech.2008.082792

10. Choi H, Rauh V, Garfinkel R, Tu Y, Perera FP. Prenatal exposure to airborne polycyclic aromatic hydrocarbons and risk of intrauterine growth restriction. Environ Health Perspect (2008) 116:658-65. doi:10.1289/ehp.10958

11. Meecham JG, Thomson CW, Blair GS. A model for investigating the systemic effects of adrenaline in dental local anaesthetic solutions in patients under surgical stress. SAAD Dig (1991) 8:19-25.

12. Brown RC, Lockwood AH, Sonawane BR. Neurodegenerative diseases: an overview of environmental risk factors. Environ Health Perspect (2005) 113:1250-6. doi:10.1289/ehp.7567

13. Fombonne E. Epidemiology of pervasive developmental disorders. Pediatr Res (2009) 65:591-8. doi:10.1203/PDR.0b013e31819e7203 determine susceptibility will provide better ways to characterize susceptible groups within the population and guide to develop better health policies to protect the population. Finally, positive results from this effort could potentially lead to revision of current limits for the primary national ambient air quality standards for criteria pollutants to be set low enough to protect the health of all susceptible groups within the population and consequently contributes to meeting the requirement of the U.S. Clean Air Act (127).

\section{AUTHOR CONTRIBUTIONS}

Ms. RB, Dr. XX, and Dr. SH are responsible for literature review and manuscript drafting.

\section{FUNDING}

This project was supported by National Institute of Environmental Health Sciences Career Development Award (\#1K01ES019177-01).

14. Fox MA, Tran NL, Groopman JD, Burke TA. Toxicological resources for cumulative risk: an example with hazardous air pollutants. Regul Toxicol Pharmacol (2004) 40:305-11. doi:10.1016/j.yrtph.2004.07.008

15. Pajovic SB, Saicic ZS, Spasic MB, Petrovic VM. The effect of ovarian hormones on antioxidant enzyme activities in the brain of male rats. Physiol Res (2003) 52:189-94.

16. Landrigan PJ, Sonawane B, Butler RN, Trasande L, Callan R, Droller D. Early environmental origins of neurodegenerative disease in later life. Environ Health Perspect (2005) 113:1230-3. doi:10.1289/ehp.7571

17. Moller P, Jacobsen NR, Folkmann JK, Danielsen PH, Mikkelsen L, Hemmingsen JG, et al. Role of oxidative damage in toxicity of particulates. Free Radic Res (2010) 44:1-46. doi:10.3109/10715760903300691

18. Hassing HC, Twickler TB, Kastelein JJ, Cramer MJ, Cassee FR. Air pollution as noxious environmental factor in the development of cardiovascular disease. Neth J Med (2009) 67:116-21.

19. Kipen H, Rich D, Huang W, Zhu T, Wang G, Hu M, et al. Measurement of inflammation and oxidative stress following drastic changes in air pollution during the Beijing Olympics: a panel study approach. Ann N Y Acad Sci (2010) 1203:160-7. doi:10.1111/j.1749-6632.2010.05638.x

20. Mills NL, Donaldson K, Hadoke PW, Boon NA, MacNee W, Cassee FR, et al. Adverse cardiovascular effects of air pollution. Nat Clin Pract Cardiovasc Med (2009) 6:36-44. doi:10.1038/ncpcardio1399

21. Oberdorster G, Utell MJ. Ultrafine particles in the urban air: to the respiratory tract - and beyond? Environ Health Perspect (2002) 110:A440-1. doi:10.1289/ ehp.110-a440

22. Calderón-Garcidueñas L, Franco-Lira M, Henriquez-Roldan C, Osnaya N, Gonzalez-Maciel A, Reynoso-Robles R, et al. Urban air pollution: influences on olfactory function and pathology in exposed children and young adults. Exp Toxicol Pathol (2010) 62:91-102. doi:10.1016/j.etp.2009.02.117

23. Calderón-Garcidueñas L, Mora-Tiscareno A, Ontiveros E, Gomez-Garza G, Barragan-Mejia G, Broadway J, et al. Air pollution, cognitive deficits and brain abnormalities: a pilot study with children and dogs. Brain $\operatorname{Cogn}$ (2008) 68:117-27. doi:10.1016/j.bandc.2008.04.008

24. Calderón-Garcidueñas L, Reed W, Maronpot RR, Henriquez-Roldan C, Delgado-Chavez R, Calderón-Garcidueñas A, et al. Brain inflammation and Alzheimer's-like pathology in individuals exposed to severe air pollution. Toxicol Pathol (2004) 32:650-8. doi:10.1080/01926230490520232

25. Cruts B, van Etten L, Tornqvist H, Blomberg A, Sandstrom T, Mills NL, et al. Exposure to diesel exhaust induces changes in EEG in human volunteers. Part Fibre Toxicol (2008) 5:4. doi:10.1186/1743-8977-5-4

26. Calderón-Garcidueñas L, Serrano-Sierra A, Torres-Jardon R, Zhu H, Yuan Y, Smith D, et al. The impact of environmental metals in young 
urbanites' brains. Exp Toxicol Pathol (2013) 65(5):503-11. doi:10.1016/j. etp. 2012.02 .006

27. Cliff R, Curran J, Hirota JA, Brauer M, Feldman H, Carlsten C. Effect of diesel exhaust inhalation on blood markers of inflammation and neurotoxicity: a controlled, blinded crossover study. Inhal Toxicol (2016) 28:145-53. doi:10.3 109/08958378.2016.1145770

28. Ailshire JA, Clarke P. Fine particulate matter air pollution and cognitive function among U.S. older adults. J Gerontol B Psychol Sci Soc Sci (2015) 70:322-8. doi:10.1093/geronb/gbu064

29. Ailshire JA, Crimmins EM. Fine particulate matter air pollution and cognitive function among older US adults. Am J Epidemiol (2014) 180:359-66. doi:10.1093/aje/kwu155

30. Bowler RM, Kornblith ES, Gocheva VV, Colledge MA, Bollweg G, Kim $\mathrm{Y}$, et al. Environmental exposure to manganese in air: associations with cognitive functions. Neurotoxicology (2015) 49:139-48. doi:10.1016/j. neuro.2015.06.004

31. Chen JC, Schwartz J. Neurobehavioral effects of ambient air pollution on cognitive performance in US adults. Neurotoxicology (2009) 30:231-9. doi:10.1016/j.neuro.2008.12.011

32. Gatto NM, Henderson VW, Hodis HN, St John JA, Lurmann F, Chen JC, et al. Components of air pollution and cognitive function in middle-aged and older adults in Los Angeles. Neurotoxicology (2014) 40:1-7. doi:10.1016/j. neuro.2013.09.004

33. Inserra SG, Phifer BL, Anger WK, Lewin M, Hilsdon R, White MC. Neurobehavioral evaluation for a community with chronic exposure to hydrogen sulfide gas. Environ Res (2004) 95:53-61. doi:10.1016/j. envres.2003.08.005

34. Loop MS, Kent ST, Al-Hamdan MZ, Crosson WL, Estes SM, Estes MG Jr, et al. Fine particulate matter and incident cognitive impairment in the reasons for geographic and racial differences in stroke (REGARDS) cohort. PLoS One (2013) 8:e75001. doi:10.1371/journal.pone.0075001

35. Power MC, Weisskopf MG, Alexeeff SE, Coull BA, Spiro A III, Schwartz J. Traffic-related air pollution and cognitive function in a cohort of older men. Environ Health Perspect (2011) 119:682-7. doi:10.1289/ehp.1002767

36. Ranft U, Schikowski T, Sugiri D, Krutmann J, Kramer U. Long-term exposure to traffic-related particulate matter impairs cognitive function in the elderly. Environ Res (2009) 109:1004-11. doi:10.1016/j.envres.2009.08.003

37. Tonne C, Elbaz A, Beevers S, Singh-Manoux A. Traffic-related air pollution in relation to cognitive function in older adults. Epidemiology (2014) 25:674-81. doi:10.1097/EDE.0000000000000144

38. Tzivian L, Dlugaj M, Winkler A, Weinmayr G, Hennig F, Fuks KB, et al. Long-term air pollution and traffic noise exposures and mild cognitive impairment in older adults: a cross-sectional analysis of the Heinz Nixdorf recall study. Environ Health Perspect (2016). doi:10.1289/ ehp. 1509824

39. Wellenius GA, Boyle LD, Coull BA, Milberg WP, Gryparis A, Schwartz J, et al. Residential proximity to nearest major roadway and cognitive function in community-dwelling seniors: results from the MOBILIZE Boston study. J Am Geriatr Soc (2012) 60:2075-80. doi:10.1111/j.1532-5415.2012.04195.x

40. Weuve J, Puett RC, Schwartz J, Yanosky JD, Laden F, Grodstein F. Exposure to particulate air pollution and cognitive decline in older women. Arch Intern Med (2012) 172:219-27. doi:10.1001/archinternmed.2011.683

41. Angelici L, Piola M, Cavalleri T, Randi G, Cortini F, Bergamaschi R, et al. Effects of particulate matter exposure on multiple sclerosis hospital admission in Lombardy Region, Italy. Environ Res (2016) 145:68-73. doi:10.1016/j. envres.2015.11.017

42. Chang KH, Chang MY, Muo CH, Wu TN, Chen CY, Kao CH. Increased risk of dementia in patients exposed to nitrogen dioxide and carbon monoxide: a population-based retrospective cohort study. PLoS One (2014) 9:e103078. doi:10.1371/journal.pone. 0103078

43. Finkelstein MM, Jerrett M. A study of the relationships between Parkinson's disease and markers of traffic-derived and environmental manganese air pollution in two Canadian cities. Environ Res (2007) 104:420-32. doi:10.1016/j. envres.2007.03.002

44. Heydarpour P, Amini H, Khoshkish S, Seidkhani H, Sahraian MA, Yunesian M. Potential impact of air pollution on multiple sclerosis in Tehran, Iran. Neuroepidemiology (2014) 43:233-8. doi:10.1159/000368553
45. Jung CR, Lin YT, Hwang BF. Ozone, particulate matter, and newly diagnosed Alzheimer's disease: a population-based cohort study in Taiwan. J Alzheimers Dis (2015) 44:573-84. doi:10.3233/JAD-140855

46. Malek AM, Barchowsky A, Bowser R, Heiman-Patterson T, Lacomis D, Rana $\mathrm{S}$, et al. Exposure to hazardous air pollutants and the risk of amyotrophic lateral sclerosis. Environ Pollut (2015) 197:181-6. doi:10.1016/j.envpol.2014.12.010

47. Oudin A, Forsberg B, Adolfsson AN, Lind N, Modig L, Nordin M, et al. Traffic-related air pollution and dementia incidence in Northern Sweden: a longitudinal study. Environ Health Perspect (2016) 124:306-12. doi:10.1289/ ehp. 1408322

48. Apte MG, Buchanan IS, Mendell MJ. Outdoor ozone and building-related symptoms in the BASE study. Indoor Air (2008) 18:156-70. doi:10.1111/j.1600-0668.2008.00521.x

49. Calderón-Garcidueñas L, Reynoso-Robles R, Vargas-Martinez J, GomezMaqueo-Chew A, Perez-Guille B, Mukherjee PS, et al. Prefrontal white matter pathology in air pollution exposed Mexico City young urbanites and their potential impact on neurovascular unit dysfunction and the development of Alzheimer's disease. Environ Res (2016) 146:404-17. doi:10.1016/j. envres.2015.12.031

50. Calderón-Garcidueñas L, Engle R, Mora-Tiscareno A, Styner M, GomezGarza G, Zhu H, et al. Exposure to severe urban air pollution influences cognitive outcomes, brain volume and systemic inflammation in clinically healthy children. Brain Cogn (2011) 77:345-55. doi:10.1016/j.bandc.2011.09.006

51. Clark C, Crombie R, Head J, van Kamp I, van Kempen E, Stansfeld SA. Does traffic-related air pollution explain associations of aircraft and road traffic noise exposure on children's health and cognition? A secondary analysis of the United Kingdom sample from the RANCH project. Am J Epidemiol (2012) 176:327-37. doi:10.1093/aje/kws012

52. Forns J, Dadvand P, Foraster M, Alvarez-Pedrerol M, Rivas I, Lopez-Vicente $\mathrm{M}$, et al. Traffic-related air pollution, noise at school, and behavioral problems in Barcelona schoolchildren: a cross-sectional study. Environ Health Perspect (2016) 124:529-35. doi:10.1289/ehp.1409449

53. Freire C, Ramos R, Puertas R, Lopez-Espinosa MJ, Julvez J, Aguilera I, et al. Association of traffic-related air pollution with cognitive development in children. JEpidemiol Community Health (2010) 64:223-8. doi:10.1136/ jech.2008.084574

54. Grineski SE, Clark-Reyna SE, Collins TW. School-based exposure to hazardous air pollutants and grade point average: a multi-level study. Environ Res (2016) 147:164-71. doi:10.1016/j.envres.2016.02.004

55. Harris MH, Gold DR, Rifas-Shiman SL, Melly SJ, Zanobetti A, Coull BA, et al. Prenatal and childhood traffic-related pollution exposure and childhood cognition in the project viva cohort (Massachusetts, USA). Environ Health Perspect (2015) 123:1072-8. doi:10.1289/ehp.1408803

56. Kicinski M, Vermeir G, Van Larebeke N, Den Hond E, Schoeters G, Bruckers L, et al. Neurobehavioral performance in adolescents is inversely associated with traffic exposure. Environ Int (2015) 75:136-43. doi:10.1016/j. envint.2014.10.028

57. Lin CC, Yang SK, Lin KC, Ho WC, Hsieh WS, Shu BC, et al. Multilevel analysis of air pollution and early childhood neurobehavioral development. Int J Environ Res Public Health (2014) 11:6827-41. doi:10.3390/ ijerph110706827

58. Suglia SF, Gryparis A, Wright RO, Schwartz J, Wright RJ. Association of black carbon with cognition among children in a prospective birth cohort study. Am J Epidemiol (2008) 167:280-6. doi:10.1093/aje/kwm308

59. Sunyer J, Esnaola M, Alvarez-Pedrerol M, Forns J, Rivas I, Lopez-Vicente M, et al. Association between traffic-related air pollution in schools and cognitive development in primary school children: a prospective cohort study. PLoS Med (2015) 12:e1001792. doi:10.1371/journal.pmed.1001792

60. van Kempen E, Fischer P, Janssen N, Houthuijs D, van Kamp I, Stansfeld $S$, et al. Neurobehavioral effects of exposure to traffic-related air pollution and transportation noise in primary schoolchildren. Environ Res (2012) 115:18-25. doi:10.1016/j.envres.2012.03.002

61. Wang S, Zhang J, Zeng X, Zeng Y, Chen S. Association of traffic-related air pollution with children's neurobehavioral functions in Quanzhou, China. Environ Health Perspect (2009) 117:1612-8. doi:10.1289/ehp.0800023

62. Chiu YH, Hsu HH, Coull BA, Bellinger DC, Kloog I, Schwartz J, et al. Prenatal particulate air pollution and neurodevelopment in urban children: 
examining sensitive windows and sex-specific associations. Environ Int (2016) 87:56-65. doi:10.1016/j.envint.2015.11.010

63. Cowell WJ, Bellinger DC, Coull BA, Gennings C, Wright RO, Wright RJ. Associations between prenatal exposure to black carbon and memory domains in Urban children: modification by sex and prenatal stress. PLoS One (2015) 10:e0142492. doi:10.1371/journal.pone.0142492

64. Guxens M, Aguilera I, Ballester F, Estarlich M, Fernandez-Somoano A, Lertxundi A, et al. Prenatal exposure to residential air pollution and infant mental development: modulation by antioxidants and detoxification factors. Environ Health Perspect (2012) 120(1):144-9. doi:10.1289/ehp.1103469

65. Guxens M, Garcia-Esteban R, Giorgis-Allemand L, Forns J, Badaloni C, Ballester F, et al. Air pollution during pregnancy and childhood cognitive and psychomotor development: six European birth cohorts. Epidemiology (2014) 25:636-47. doi:10.1097/EDE.0000000000000133

66. Jedrychowski WA, Perera FP, Camann D, Spengler J, Butscher M, Mroz E, et al. Prenatal exposure to polycyclic aromatic hydrocarbons and cognitive dysfunction in children. Environ Sci Pollut Res Int (2015) 22:3631-9. doi:10.1007/s11356-014-3627-8

67. Kim E, Park H, Hong YC, Ha M, Kim Y, Kim BN, et al. Prenatal exposure to $\mathrm{PM}(1)(0)$ and $\mathrm{NO}(2)$ and children's neurodevelopment from birth to 24 months of age: mothers and children's environmental health (MOCEH) study. Sci Total Environ (2014) 481:439-45. doi:10.1016/j.scitotenv.2014.01.107

68. Lovasi GS, Eldred-Skemp N, Quinn JW, Chang HW, Rauh VA, Rundle A, et al. Neighborhood social context and individual polycyclic aromatic hydrocarbon exposures associated with child cognitive test scores. J Child Fam Stud (2014) 23:785-99. doi:10.1007/s10826-013-9731-4

69. Perera FP, Rauh V, Whyatt RM, Tsai WY, Tang D, Diaz D, et al. Effect of prenatal exposure to airborne polycyclic aromatic hydrocarbons on neurodevelopment in the first 3 years of life among inner-city children. Environ Health Perspect (2006) 114:1287-92. doi:10.1289/ehp.9084

70. Porta D, Narduzzi S, Badaloni C, Bucci S, Cesaroni G, Colelli V, et al. Air pollution and cognitive development at age 7 in a prospective Italian birth cohort. Epidemiology (2016) 27:228-36. doi:10.1097/ EDE.0000000000000405

71. Tang D, Li TY, Liu JJ, Zhou ZJ, Yuan T, Chen YH, et al. Effects of prenatal exposure to coal-burning pollutants on children's development in China. Environ Health Perspect (2008) 116:674-9. doi:10.1289/ehp.10471

72. Yorifuji T, Kashima S, Higa Diez M, Kado Y, Sanada S, Doi H. Prenatal exposure to traffic-related air pollution and child behavioral development milestone delays in Japan. Epidemiology (2016) 27:57-65. doi:10.1097/ EDE.0000000000000361

73. Siddique S, Banerjee M, Ray MR, Lahiri T. Attention-deficit hyperactivity disorder in children chronically exposed to high level of vehicular pollution. Eur J Pediatr (2011) 170:923-9. doi:10.1007/s00431-010-1379-0

74. Newman NC, Ryan P, Lemasters G, Levin L, Bernstein D, Hershey GK, et al. Traffic-related air pollution exposure in the first year of life and behavioral scores at 7 years of age. Environ Health Perspect (2013) 121:731-6. doi:10.1289/ehp.1205555

75. Gong T, Almqvist C, Bolte S, Lichtenstein P, Anckarsater H, Lind T, et al. Exposure to air pollution from traffic and neurodevelopmental disorders in Swedish twins. Twin Res Hum Genet (2014) 17:553-62. doi:10.1017/ thg. 2014.58

76. Windham GC, Zhang L, Gunier R, Croen LA, Grether JK. Autism spectrum disorders in relation to distribution of hazardous air pollutants in the San Francisco Bay Area. Environ Health Perspect (2006) 114:1438-44. doi:10.1289/ehp. 9120

77. Kalkbrenner AE, Daniels JL, Chen JC, Poole C, Emch M, Morrissey J. Perinatal exposure to hazardous air pollutants and autism spectrum disorders at age 8 . Epidemiology (2010) 21:631-41. doi:10.1097/EDE.0b013e3181e65d76

78. Volk HE, Hertz-Picciotto I, Delwiche L, Lurmann F, McConnell R. Residential proximity to freeways and autism in the CHARGE study. Environ Health Perspect (2011) 119:873-7. doi:10.1289/ehp.1002835

79. Becerra TA, Wilhelm M, Olsen J, Cockburn M, Ritz B. Ambient air pollution and autism in Los Angeles County, California. Environ Health Perspect (2013) 121:380-6. doi:10.1289/ehp.1205827

80. Jung CR, Lin YT, Hwang BF. Air pollution and newly diagnostic autism spectrum disorders: a population-based cohort study in Taiwan. PLoS One (2013) 8:e75510. doi:10.1371/journal.pone.0075510
81. Roberts AL, Lyall K, Hart JE, Laden F, Just AC, Bobb JF, et al. Perinatal air pollutant exposures and autism spectrum disorder in the children of nurses' health study ii participants. Environ Health Perspect (2013) 121:978-84. doi:10.1289/ehp.1206187

82. Volk HE, Lurmann F, Penfold B, Hertz-Picciotto I, McConnell R. Trafficrelated air pollution, particulate matter, and autism. JAMA Psychiatry (2013) 70:71-7. doi:10.1001/jamapsychiatry.2013.266

83. Kalkbrenner AE, Windham GC, Serre ML, Akita Y, Wang X, Hoffman K, et al. Particulate matter exposure, prenatal and postnatal windows of susceptibility, and autism spectrum disorders. Epidemiology (2015) 26:30-42. doi:10.1097/EDE.0000000000000173

84. Raz R, Roberts AL, Lyall K, Hart JE, Just AC, Laden F, et al. Autism spectrum disorder and particulate matter air pollution before, during, and after pregnancy: a nested case-control analysis within the nurses' health study II cohort. Environ Health Perspect (2015) 123:264-70. doi:10.1289/ ehp. 1408133

85. Talbott EO, Arena VC, Rager JR, Clougherty JE, Michanowicz DR, Sharma RK, et al. Fine particulate matter and the risk of autism spectrum disorder. Environ Res (2015) 140:414-20. doi:10.1016/j.envres.2015.04.021

86. Talbott EO, Marshall LP, Rager JR, Arena VC, Sharma RK, Stacy SL. Air toxics and the risk of autism spectrum disorder: the results of a population based case-control study in southwestern Pennsylvania. Environ Health (2015) 14:80. doi:10.1186/s12940-015-0064-1

87. Guxens M, Ghassabian A, Gong T, Garcia-Esteban R, Porta D, GiorgisAllemand L, et al. Air pollution exposure during pregnancy and childhood autistic traits in four European population-based cohort studies: the ESCAPE project. Environ Health Perspect (2016) 124:133-40. doi:10.1289/ehp.1408483

88. Bolton JL, Smith SH, Huff NC, Gilmour MI, Foster WM, Auten RL, et al. Prenatal air pollution exposure induces neuroinflammation and predisposes offspring to weight gain in adulthood in a sex-specific manner. FASEB $J$ (2012) 26:4743-54. doi:10.1096/f.12-210989

89. Calderón-Garcidueñas L, Mora-Tiscareno A, Gomez-Garza G, CarrascoPortugal Mdel C, Perez-Guille B, Flores-Murrieta FJ, et al. Effects of a cyclooxygenase-2 preferential inhibitor in young healthy dogs exposed to air pollution: a pilot study. Toxicol Pathol (2009) 37:644-60. doi:10.1177/0192623309340277

90. Levesque S, Surace MJ, McDonald J, Block ML. Air pollution \& the brain: subchronic diesel exhaust exposure causes neuroinflammation and elevates early markers of neurodegenerative disease. J Neuroinflammation (2011) 8:105. doi:10.1186/1742-2094-8-105

91. Levesque S, Taetzsch T, Lull ME, Kodavanti U, Stadler K, Wagner A, et al. Diesel exhaust activates and primes microglia: air pollution, neuroinflammation, and regulation of dopaminergic neurotoxicity. Environ Health Perspect (2011) 119:1149-55. doi:10.1289/ehp.1002986

92. Miranda ML, Edwards SE, Keating MH, Paul CJ. Making the environmental justice grade: the relative burden of air pollution exposure in the United States. Int J Environ Res Public Health (2011) 8:1755-71. doi:10.3390/ijerph8061755

93. Romieu I, Moreno-Macias H, London SJ. Gene by environment interaction and ambient air pollution. Proc Am Thorac Soc (2010) 7:116-22. doi:10.1513/ pats.200909-097RM

94. Gupta RC, Reddy MV, Randerath K. 32P-postlabeling analysis of non-radioactive aromatic carcinogen - DNA adducts. Carcinogenesis (1982) 3:1081-92. doi:10.1093/carcin/3.9.1081

95. Sram RJ, Binkova B. Molecular epidemiology studies on occupational and environmental exposure to mutagens and carcinogens, 1997-1999. Environ Health Perspect (2000) 108(Suppl 1):57-70. doi:10.1289/ehp.00108s157

96. Nielsen PS, de Pater N, Okkels H, Autrup H. Environmental air pollution and DNA adducts in Copenhagen bus drivers - effect of GSTM1 and NAT2 genotypes on adduct levels. Carcinogenesis (1996) 17:1021-7. doi:10.1093/ carcin/17.5.1021

97. Binkova B, Lewtas J, Miskova I, Lenicek J, Sram R. DNA adducts and personal air monitoring of carcinogenic polycyclic aromatic hydrocarbons in an environmentally exposed population. Carcinogenesis (1995) 16:1037-46. doi:10.1093/carcin/16.5.1037

98. Autrup H, Daneshvar B, Dragsted LO, Gamborg M, Hansen M, Loft S, et al. Biomarkers for exposure to ambient air pollution - comparison of carcinogenDNA adduct levels with other exposure markers and markers for oxidative stress. Environ Health Perspect (1999) 107:233-8. doi:10.2307/3434514 
99. Choi H, Jedrychowski W, Spengler J, Camann DE, Whyatt RM, Rauh V, et al. International studies of prenatal exposure to polycyclic aromatic hydrocarbons and fetal growth. Environ Health Perspect (2006) 114:1744-50.

100. Jedrychowski WA, Perera FP, Maugeri U, Mroz E, Klimaszewska-Rembiasz M, Flak E, et al. Effect of prenatal exposure to fine particulate matter on ventilatory lung function of preschool children of non-smoking mothers. Paediatr Perinat Epidemiol (2010) 24:492-501. doi:10.1111/j.1365-3016.2010. 01136.x

101. Briggs DJ, de Hoogh C, Gulliver J, Wills J, Elliott P, Kingham S, et al. A regression-based method for mapping traffic-related air pollution: application and testing in four contrasting urban environments. Sci Total Environ (2000) 253:151-67. doi:10.1016/S0048-9697(00)00429-0

102. Gilbert NL, Goldberg MS, Beckerman B, Brook JR, Jerrett M. Assessing spatial variability of ambient nitrogen dioxide in Montreal, Canada, with a land-use regression model. J Air Waste Manag Assoc (2005) 55:1059-63. doi:10.1080/10473289.2005.10464708

103. Sahsuvaroglu T, Arain A, Kanaroglou P, Finkelstein N, Newbold B, Jerrett $\mathrm{M}$, et al. A land use regression model for predicting ambient concentrations of nitrogen dioxide in Hamilton, Ontario, Canada. J Air Waste Manag Assoc (2006) 56:1059-69. doi:10.1080/10473289.2006.10464542

104. Brauer M, Hoek G, Smit HA, de Jongste JC, Gerritsen J, Postma DS, et al. Air pollution and development of asthma, allergy and infections in a birth cohort. Eur Respir J (2007) 29:879-88. doi:10.1183/09031936. 00083406

105. Ryan PH, LeMasters GK. A review of land-use regression models for characterizing intraurban air pollution exposure. Inhal Toxicol (2007) 19(Suppl 1):127-33. doi:10.1080/08958370701495998

106. Ben-Shlomo Y, Kuh D. A life course approach to chronic disease epidemiology: conceptual models, empirical challenges and interdisciplinary perspectives. Int J Epidemiol (2002) 31:285-93. doi:10.1093/ije/31.2.285

107. Laumbach RJ, Kipen HM. Acute effects of motor vehicle traffic-related air pollution exposures on measures of oxidative stress in human airways. Ann N Y Acad Sci (2011) 1203:107-12. doi:10.1111/j.1749-6632.2010. 05604.x

108. Tao Y, Zhong L, Huang X, Lu SE, Li Y, Dai L, et al. Acute mortality effects of carbon monoxide in the Pearl River Delta of China. Sci Total Environ (2011) 410:34-40. doi:10.1016/j.scitotenv.2011.09.004

109. Ferreira-Ceccato AD, Ramos EM, de Carvalho LC Jr, Xavier RF, Teixeira MF, Raymundo-Pereira PA, et al. Short terms effects of air pollution from biomass burning in mucociliary clearance of Brazilian sugarcane cutters. Respir Med (2011) 105:1766-8. doi:10.1016/j.rmed.2011.08.003

110. Menezes-Filho JA, Bouchard M, Sarcinelli Pde N, Moreira JC. Manganese exposure and the neuropsychological effect on children and adolescents: a review. Rev Panam Salud Publica (2009) 26:541-8. doi:10.1590/ S1020-49892009001200010

111. Sanders T, Liu Y, Buchner V, Tchounwou PB. Neurotoxic effects and biomarkers of lead exposure: a review. Rev Environ Health (2009) 24:15-45. doi:10.1515/REVEH.2009.24.1.15

112. Winneke G, Walkowiak J, Lilienthal H. PCB-induced neurodevelopmental toxicity in human infants and its potential mediation by endocrine dysfunction. Toxicology (2002) 18(1-182):161-5. doi:10.1016/S0300-483X(02) 00274-3

113. Jurewicz J, Hanke W. Prenatal and childhood exposure to pesticides and neurobehavioral development: review of epidemiological studies. Int J Occup Med Environ Health (2008) 21:121-32. doi:10.2478/v10001-008-0014-z
114. American Academy of Pediatrics Committee on Environmental Health. Lead exposure in children: prevention, detection, and management. Pediatrics (2005) 116:1036-46. doi:10.1542/peds.2005-1947

115. Sabia S, Marmot M, Dufouil C, Singh-Manoux A. Smoking history and cognitive function in middle age from the Whitehall II study. Arch Intern Med (2008) 168:1165-73. doi:10.1001/archinte.168.11.1165

116. Stephens R, Ling J, Heffernan TM, Heather N, Jones K. A review of the literature on the cognitive effects of alcohol hangover. Alcohol Alcohol (2008) 43:163-70. doi:10.1093/alcalc/agm 160

117. Llewellyn DJ, Lang IA, Langa KM, Naughton F, Matthews FE. Exposure to secondhand smoke and cognitive impairment in non-smokers: national cross sectional study with cotinine measurement. BMJ (2009) 338:b462. doi:10.1136/bmj.b462

118. Ott A, Andersen K, Dewey ME, Letenneur L, Brayne C, Copeland JR, et al. Effect of smoking on global cognitive function in nondemented elderly. Neurology (2004) 62:920-4. doi:10.1212/01.WNL.0000115110.35610.80

119. Weitzman M, Gortmaker S, Sobol A. Maternal smoking and behavior problems of children. Pediatrics (1992) 90:342-9.

120. Julvez J, Ribas-Fito N, Torrent M, Forns M, Garcia-Esteban R, Sunyer J. Maternal smoking habits and cognitive development of children at age 4 years in a population-based birth cohort. Int J Epidemiol (2007) 36:825-32. doi:10.1093/ije/dym 107

121. Ngandu T, Helkala EL, Soininen H, Winblad B, Tuomilehto J, Nissinen A, et al. Alcohol drinking and cognitive functions: findings from the cardiovascular risk factors aging and dementia (CAIDE) study. Dement Geriatr Cogn Disord (2007) 23:140-9. doi:10.1159/000097995

122. Solfrizzi V, D'Introno A, Colacicco AM, Capurso C, Del Parigi A, Baldassarre G, et al. Alcohol consumption, mild cognitive impairment, and progression to dementia. Neurology (2007) 68:1790-9. doi:10.1212/01. wnl.0000262035.87304.89

123. Townsend MK, Devore E, Kang JH, Grodstein F. The relation between moderate alcohol consumption and cognitive function in older women with type 2 diabetes. Diabetes Res Clin Pract (2009) 85:322-7. doi:10.1016/j. diabres.2009.06.023

124. Stampfer MJ, Kang JH, Chen J, Cherry R, Grodstein F. Effects of moderate alcohol consumption on cognitive function in women. N Engl J Med (2005) 352:245-53. doi:10.1056/NEJMoa041152

125. Siddiqi N. Publication bias in epidemiological studies. Cent Eur J Public Health (2011) 19:118-20.

126. Song F, Parekh S, Hooper L, Loke YK, Ryder J, Sutton AJ, et al. Dissemination and publication of research findings: an updated review of related biases. Health Technol Assess (2011) 14:iii,ix-xi,1-193.

127. Kleeberger SR. Genetic aspects of susceptibility to air pollution. Eur Respir J Suppl (2003) 40:52s-6s. doi:10.1183/09031936.03.00403003

Conflict of Interest Statement: The authors declare that the research was conducted in the absence of any commercial or financial relationships that could be construed as a potential conflict of interest.

Copyright ( $\odot 2016 \mathrm{Xu}, \mathrm{Ha}$ and Basnet. This is an open-access article distributed under the terms of the Creative Commons Attribution License (CC BY). The use, distribution or reproduction in other forums is permitted, provided the original author(s) or licensor are credited and that the original publication in this journal is cited, in accordance with accepted academic practice. No use, distribution or reproduction is permitted which does not comply with these terms. 